Mens

Revue d'histoire intellectuelle et culturelle

mens

\title{
La contribution de la Francophonie mondiale au démantèlement du Canada français ou l'évolution du mouvement Richelieu (1944-1982)
}

\section{Serge Dupuis}

Volume 14, numéro 1, automne 2013

URI : https://id.erudit.org/iderudit/1032623ar

DOI : https://doi.org/10.7202/1032623ar

Aller au sommaire du numéro

Éditeur(s)

Centre de recherche en civilisation canadienne-française

ISSN

1492-8647 (imprimé)

1927-9299 (numérique)

Découvrir la revue

Citer cet article

Dupuis, S. (2013). La contribution de la Francophonie mondiale au démantèlement du Canada français ou l'évolution du mouvement Richelieu (1944-1982). Mens, 14(1), 91-138. https://doi.org/10.7202/1032623ar
Résumé de l'article

Cet article souligne la contribution de la Francophonie mondiale au démantèlement du projet national canadien-français entre les années 1950 et 1980. L'auteur reconnaît que le projet canadien-français continental s'est progressivement effrité non seulement à cause du ralentissement des migrations hors Québec, de l'intervention croissante de l'État, du néonationalisme québécois et du fédéralisme renouvelé, mais aussi parce que le projet mondial émergent de la Francophonie a, pendant ces mêmes années, marqué l'esprit des Canadiens français. L'évolution du mouvement Richelieu en constitue un parfait exemple. La mutation d'un organisme

canadien-français en organisme francophone mondial à partir de la fin de la décennie 1960 rappelle dans quelle mesure ce nouvel échelon de solidarité a rassemblé des gens de différents continents, qui se sont progressivement ralliés à un projet et à une identité « francophones » au détriment d’un projet national plus restreint comme celui du Canada français. 


\title{
La contribution de la Francophonie mondiale au démantèlement du Canada français ou l'évolution du mouvement Richelieu (1944-1982)
}

\author{
Serge Dupuis \\ Université Laval
}

\section{Résumé}

Cet article souligne la contribution de la Francophonie mondiale au démantèlement du projet national canadien-français entre les années 1950 et 1980. L'auteur reconnaît que le projet canadien-français continental s'est progressivement effrité non seulement à cause du ralentissement des migrations hors Québec, de l'intervention croissante de l'État, du néonationalisme québécois et du fédéralisme renouvelé, mais aussi parce que le projet mondial émergent de la Francophonie a, pendant ces mêmes années, marqué l'esprit des Canadiens français. L'évolution du mouvement Richelieu en constitue un parfait exemple. La mutation d'un organisme canadien-français en organisme francophone mondial à partir de la fin de la décennie 1960 rappelle dans quelle mesure ce nouvel échelon de solidarité a rassemblé des gens de différents continents, qui se sont progressivement ralliés à un projet et à une identité « francophones » au détriment d'un projet national plus restreint comme celui du Canada français.

\section{Abstract}

This article underlines the contribution of the Francophonie towards the dismantlement of the French Canadian national project between the 1950s and the 1980s. The author recognizes that the French Canadian 
continental project was progressively eroded by the slowing of migrations outside Quebec, the increasing interventions of the State, Quebec neonationalism, and the renewal of federalism, but also suggests that the emerging Francophonie project, during these years, also had an impact on the minds of French Canadians. The Richelieu movement's evolution constitutes a perfect example of this tendency. Its transformation from a French Canadian to a Francophone and international movement the late 1960s shows the way in which this new level of solidarity brought various individuals on different continents to rally towards and identify with what was considered "francophone", to the detriment of a more restrictive national project such as French Canada.

Certains diraient que le démantèlement du Canada français fait presque l'objet d'une obsession dans l'historiographie contemporaine. Les déchirements idéologiques et institutionnels qu'a apportés la décennie 1960 laissent toujours des séquelles chez les intellectuels francophones des milieux minoritaires qui les ont vécus, alors que les plus jeunes tendent à être fascinés par cette époque où les minorités canadiennes-françaises occupaient une place centrale dans le discours sur l'avenir du Québec. On continue de s'interroger sur les tenants et les aboutissants de la métamorphose de la question nationale non seulement parce qu' « une partie du Canada français [...] a été coupée ${ }^{1}$ " pendant ces années, pour reprendre l'expression du sociologue Jacques Beauchemin, mais aussi parce que ce projet inspire toujours un certain " respect ", selon les sociologues Joseph Yvon Thériault et E.-Martin Meunier², comme unique projet de

1 Jean-François Laniel, "Le Canada français et nous : entretien avec Jacques Beauchemin ", La Relève : le journal des étudiants de la francophonie canadienne, vol. 3, no 1 (hiver 2012), p. 22, [En ligne], [http://journallareleve.com/wordpress/ wp-content/uploads/2012/03/LaRelV3N1version3.pdf] (28 mai 2015).

2 Joseph Yvon Thériault et E.-Martin Meunier, "Que reste-t-il du Canada français? ", dans Joseph Yvon Thériault, Anne Gilbert et Linda Cardinal (dir.), L'espace francophone en milieu minoritaire au Canada: nouveaux enjeux, nouvelles mobilisations, Montréal, Éditions Fides, 2008, p. 205-238. 
société globale qui comprenait les minorités et qui n'était pas dicté par les aspirations d'une majorité exogène.

L'historien Marcel Martel a été l'un des premiers à se prononcer sur la « rupture " du Canada français en rappelant les nombreuses souscriptions, menées par le Québec jusqu’à la décennie 1950, pour appuyer le développement institutionnel des collectivités minoritaires ${ }^{3}$. Si c'est pendant la décennie 1960 que le Québec s'est passablement désengagé sur ce front, Yves Frenette situe la fragmentation du projet canadien-français plus en amont, pendant l'entre-deux-guerres, au moment où les migrations de masse vers la Nouvelle-Angleterre et l'Ontario ont ralenti et, par conséquent, que les rapports transfrontaliers entre proches sont devenus moins fréquents ${ }^{4}$. Les recherches plus récentes de Michel Bock, de même que celles de ses anciens étudiants, complexifient l'analyse du rapport de l'élite franco-ontarienne aux nationalistes québécois ${ }^{5}$. François-Olivier Dorais et Serge Miville soulignent, par exemple, à quel point l'élite franco-ontarienne, tout en édifiant l'autonomie institutionnelle de l'Ontario français, a voulu actualiser, dans la continuité, mais sous une nouvelle forme, la "volonté nationale " canadienne-française pendant les décennies 1970 et $1980^{6}$. Pour eux, il y aurait eu un éloignement progressif entre les aspirations des minorités et celles du Québec - dont l'impact

3 Marcel Martel, Le deuil d'un pays imaginé : rêves, luttes et déroute du Canada français: les rapports entre le Québec et la francophonie canadienne (1867-1975), Ottawa, Les Presses de l'Université d'Ottawa et le Centre de recherche en civilisation canadienne-française, 1997.

${ }^{4}$ Yves Frenette, Brève histoire des Canadiens français, avec la collaboration de Martin Pâquet, Montréal, Éditions du Boréal, 1998.

5 Michel Bock et Yves Frenette (dir.), Histoire de l'ACFO, Ottawa, Les Presses de l'Université d'Ottawa. À paraître.

${ }^{6}$ François-Olivier Dorais, "Gaétan Gervais : témoin et agent d'une mutation référentielle en Ontario français ", Mens : revue d'histoire intellectuelle et culturelle, vol. 13, n 2 (printemps 2013), p. 59-99; Serge Miville, " $A$ quoi sert au Canadien français de gagner l'univers canadien sill perd son âme de francophone?" Représentations identitaires et mémorielles dans la presse franco-ontarienne après la "rupture " du Canada français (1969-1986), thèse de maîtrise (histoire), Ottawa, Université d'Ottawa, 2012. 
des événements les plus marquants pendant la décennie 1960 aurait été exagéré -, mais pas de rupture avec l'idéal du Canada français avant que les minorités aient bénéficié de l'interprétation par les tribunaux de l'article 23 de la Charte canadienne des droits et libertés (1982) dans le domaine scolaire. C'est ainsi que le rapatriement de la Constitution et les tentatives ratées de réforme constitutionnelle (1990, 1992 et 1995) ont progressivement été perçus comme des échecs ou des victoires selon la rive de l'Outaouais que les francophones habitaient. La métamorphose idéologique et institutionnelle aurait donc commencé quelque part au milieu du $x^{\mathrm{e}}$ siècle et n'aurait été consommée qu'à la fin du millénaire, même si la référence canadienne-française n'a pas complètement disparu des projets plus récents (québécois, franco-ontarien, etc.) ni dans les nouvelles régions de migration sur le continent ou à l'étranger ${ }^{7}$.

Jamais entièrement accepté ni complètement abandonné, le projet national canadien-français risque de susciter de l'intérêt encore longtemps, d'autant plus que la décennie 1960 demeurera une période incontournable pour comprendre cette mutation. Devant la disparition de l'Ordre de Jacques-Cartier (1965), la québécisation de la Fédération des sociétés Saint-Jean-Baptiste (1966) et la marginalisation du Conseil de la vie française en Amérique, d'autres groupes politiques ont pris la relève pour défendre les intérêts nationaux des Canadiens français, dont le Parti québécois en 1968 et la Fédération des francophones hors Québec en 1976, en suivant le tracé des frontières provinciales. Par ailleurs, seuls trois organismes rassemblant le Québec et les minorités canadiennes-françaises ont survécu jusqu'à nos jours : l'Association canadienne d'enseignement en langue française (ACELF), l'Association francophone pour le savoir (ACFAS), de même que la Société Richelieu, qui est le seul des trois organismes à

\footnotetext{
Serge Dupuis, "On prévoyait le déluge! La résistance franco-ontarienne au rapatriement de la Constitution canadienne : 1977-1982 ", Revue du NouvelOntario, no 33 (2008), p. 7-39; Serge Dupuis, "Plus peur de l'hiver que du diable" : des immigrants aux hivernants canadiens-français à Palm Beach (Floride), 1945-1997 ", Revue d'histoire de l'Amérique française, vol. 63, n 4 (printemps 2010), p. 465-495.
} 
avoir maintenu un certain caractère canadien-français. En revanche, s'il a conservé un intérêt pour le Canada français - et pour le maintien de son assise organisationnelle en l'occurrence - le Richelieu l'a fait en s'arrimant simultanément au projet émergent de la Francophonie mondiale. En hissant les rapports avec les francophones d'Europe, d'Afrique et des Antilles au même rang que les relations avec les francophones de l'Amérique du Nord, le Richelieu a réussi à maintenir plusieurs de ses caractéristiques fondatrices. Cet organisme national, caritatif et masculin a été confronté aux mêmes tensions qui ont achevé, affaibli ou transformé les autres organismes patriotiques canadiens-français, mais sa réaction aux mutations de l'époque l'a distingué dans la mêlée.

Les archives du Richelieu, conservées à son siège social à Ottawa, ainsi qu'au Centre de recherche en civilisation canadienne-française de l'Université d'Ottawa, ont permis de mener cette enquête sur l'histoire du mouvement. Les archives privées de sept clubs, ainsi qu'une série d'entretiens avec 36 acteurs, ont également permis de nuancer les archives centrales du mouvement. Des récits inédits complètent également les sources écrites. Cet article s'inspire enfin d'études internes (dont celles préparées pour commémorer les $25^{\mathrm{e}}$ et $50^{\mathrm{e}}$ anniversaires du mouvement ${ }^{8}$ ), d'historiques de clubs particuliers ainsi que d'une thèse de maîtrise portant sur le cercle de Sudbury ${ }^{9}$. En trois temps, nous rappellerons le contexte entourant la fondation du Richelieu en 1944, le discours canadien-français qu’il véhiculait à ses origines, sa contribution à constituer un échelon supérieur - la Francophonie mondiale - dans la hiérarchie des projets francophones au-delà du Canada français et des projets régionaux, ainsi que les conséquences de ces efforts sur le projet canadien-français jusqu'en

${ }^{8}$ Raymond Dufresne, Les Clubs Richelieu : les premiers 25 ans du Richelieu international, Montréal, Éditions du Jour, 1971; Renée Veilleux et Céline Deschênes, Dans les sentiers de l'amitié: Richelieu international: 1944-1994, Mont-Joli, Club Richelieu Mont-Joli, 1994.

9 Brigitte Berthiaume, Le Club Richelieu Sudbury, 1947 à 1977 : le rôle des membres d'un club social dans une communauté canadienne-française, thèse de maîtrise (histoire), Sudbury, Université Laurentienne, 2009. 
1982. Le Richelieu n'a peut-être pas réussi à sauver le Canada français avec son pari de s'engager vis-à-vis la mondialisation de la Francophonie, mais il est parvenu à préserver son assise ainsi qu'un certain esprit canadien-français entre ses murs.

\section{Un nouvel organe du réseau institutionnel canadien- français (1944-1959)}

On fait souvent référence aux concepts de culture première et de culture seconde, développés par le sociologue Fernand Dumont, pour théoriser la sociologie historique particulière du Canada français entre 1840 et 1960 . En se positionnant dans le débat entre les historiens des idées, qui soulignaient les particularismes du Canada français, et les historiens du social, qui soulignaient surtout les structures socioéconomiques nord-américaines du Québec, Dumont a voulu distinguer les mours associées au travail ou au climat (la culture première) des idées relatives aux valeurs et aux aspirations collectives (la culture seconde) ${ }^{10}$. Par conséquent, si la réalité du continent a sans conteste dicté les mours des "Canadiens ", ceux-ci y ont aussi cultivé et nourri des idées françaises et catholiques distinctes de celles de la majorité anglo-protestante. C'est cette logique binaire qui semble avoir motivé l'Ordre de Jacques-Cartier (OJC) à contrecarrer l'incursion des service clubs américains au Canada français ${ }^{11}$. Ces " clubs neutres " s'étaient installés au Canada anglais et bilingue à partir des années 1910 (quelques années seulement après leur fondation aux États-Unis), mais n'ont provoqué l'ire des nationalistes canadiens-français que lorsqu'ils ont commencé à créer des clubs au Québec francophone ${ }^{12}$. On a d'abord condamné

10 Fernand Dumont, Un témoin de l'homme, entretiens colligés et présentés par Serge Cantin, Montréal, Éditions de l'Hexagone, 2000, p. 85-86.

11 Jeffrey A. Charles, Service Clubs in American Society: Rotary, Kiwanis, and Lions, Urbana, University of Illinois Press, 1993, p. 114-116.

12 "Re : Club Rotary », 11 avril 1956; «Re : Clubs neutres », 5 mai 1956, Ottawa, Bibliothèque et Archives Canada (ci-après BAC), Fonds Ordre de Jacques-Cartier (ci-après Fonds OJC), MG28-I98, vol. 147, dossier "Clubs neutres ». 
les adhésions à ces clubs, mais, n’ayant pas réussi à diminuer leur attrait chez ses compatriotes ${ }^{13}$, l'élite a cru bon de leur offrir une alternative, qui arrimerait la culture première nord-américaine aux idéaux d'une culture seconde franco-catholique...

Chancelier de l'OJC et pédiatre franco-ontarien d'Ottawa, Horace Viau a essuyé deux défaites dans sa volonté de fonder un service club canadien-français dans sa ville en 1937 et 1940. Il a donc décidé de se joindre au Rotary Club de Hull en 1941 pour y étudier les rouages et imaginer, en meilleure connaissance de cause, un nouveau «moyen d'extériorisation [de l'OJC] pour atteindre le grand public $^{14}$ ». Le 3 mai 1944, Viau a obtenu une charte sociétale fédérale pour la Société Richelieu et formé un conseil avec 17 chanceliers d'Ottawa, de Hull (Québec) et de Montréal, tous des professionnels nationalistes et commandeurs de l'OJC ${ }^{15}$. Grâce à l'appui des commanderies locales de cet organisme, le Richelieu a réussi à établir, pendant sa première décennie et demie d'existence, plus d'une centaine de cercles du Saguenay (Québec) à Fall River (Massachussetts) et de Saint-Boniface (Manitoba) à Pointe-de-l'Église (NouvelleÉcosse). En recrutant des professionnels et des gens d'affaires surtout dans de nombreuses localités de l'Amérique française, il a atteint un effectif de 6000 membres au bout d'une quinzaine d'années ${ }^{16}$. Si les cercles en milieu minoritaire n'y représentaient que $30 \%$ des clubs, l'élite d'Ottawa est pourtant demeurée majoritaire au Conseil d'administration pendant les années 1940 et $1950^{17}$. On avançait souvent que cette majorité ottavienne facilitait tout simplement, pour

13 Denise Robillard, L'Ordre de Jacques-Cartier : une société secrète pour les Canadiens français catholiques, 1926-1965, Montréal, Éditions Fides, 2009.

14 "La Société Richelieu, Charte", 3 mai 1944, BAC, Fonds OJC, MG28-I98/37/3.

15 "Première assemblée du Conseil général ", 18 janvier 1945, Ottawa, Archives privées du Richelieu International (ci-après APRI), vol. " Procès-verbaux 1944$1949 »$.

16 " Procès-verbal de la sixième assemblée annuelle », 18 février 1956, p. 2, Ottawa, Centre de recherche en civilisation canadienne-française (ci-après CRCCF), Fonds Richelieu International (ci-après Fonds RI), C76/1/6.

17 "Assemblée annuelle du Conseil général », 25 février 1950, p. 2; "Registre des administrateurs », [mars 1957], CRCCF, Fonds RI, C76/1/1 et C76/1/4. 
des questions de proximité géographique, la tenue de rencontres urgentes. Cela dit, l'organisme a retenu l'empreinte de l'élite d'Ottawa, qui a organisé les structures administratives du Richelieu sur le modèle du Canada français, avec ses minorités et ses représentations du Canada français. Par exemple, le Richelieu a refusé de former des conseils provinciaux - comme l'avaient fait l'Ordre de Jacques-Cartier et la Société Saint-Jean-Baptiste (SSJB) pendant ces années -, que l'administrateur Normand Grimard qualifiait de " provincialisme [...] de mauvais aloi ${ }^{18}$ ". Tout en véhiculant une solidarité canadiennefrançaise continentale, l'élite d'Ottawa s'est également montrée prudente devant le risque de voir la majorité du Québec rapatrier la direction du mouvement. Le rapprochement entre le foyer québécois et sa périphérie ne semblaient plus aller de soi, un indice qui laisse croire que les rapports entre les Canadiens français de divers milieux étaient peut-être déjà moins fréquents avec le ralentissement des migrations outre-frontières.

D'ailleurs, le mouvement a fondé son premier club dans la capitale fédérale en septembre 1945, mais ne l'a pas établi dans l'une ou l'autre des villes sœurs de l'Outaouais : il les a jumelées en un seul cercle. Son fondateur, le Hullois Arthur Desjardins, voyait dans le regroupement des deux villes le « symbole » de la pérennité d'une solidarité paraprovinciale.

Le club Ottawa-Hull symbolise véritablement l'union et la collaboration qui doivent exister entre toutes les branches de la famille française au Canada. Il convenait peut-être, pour cette raison, que la série des clubs Richelieu commençât à un endroit qui pût réunir à la même table les Hullois, qui sont les Québécois les plus rapprochés des Ontariens, et les Outaouais, qui sont les Ontariens les plus Québécois ${ }^{19}$.

18 Normand Grimard, dans "Le Richelieu ne souffre pas de "provincialisme" ", La Patrie, 8 novembre 1957.

19 Arthur Desjardins, "Les Clubs Richelieu : causerie à CKAC », $1^{\text {er }}$ juin 1948, dans Le Richelieu, vol. 4, n 5 (septembre 1954), p. 34, APRI, vol. «Vie Richelieu 1946-1980». 
Cette solidarité entre une région québécoise fortement marquée par l'anglais et une région ontarienne fortement marquée par le français tenait peut-être la route dans les faits, même s'il y avait, bien entendu, des distinctions entre les cercles en milieu majoritaire et ceux en milieu minoritaire quant à leurs capacités d'agir. Déjà pendant la décennie 1950, par exemple, tandis que les clubs en milieux minoritaires cherchaient seulement à ce que "la population canadienne-française » soit " considéré[e] avec dignité et respect ${ }^{20}$ " par la majorité, les clubs en milieu majoritaire justifiaient l'unilinguisme français des cercles parce qu'il représentait un moyen d'en faire la langue principale d'intégration au Québec ${ }^{21}$. Ces derniers n'hésitaient pas non plus à discuter de la nationalisation des ressources naturelles ${ }^{22}$ ni même à inviter le premier ministre Maurice Duplessis pour promouvoir l'autonomie des compétences provinciales ${ }^{23}$. En 1950, Pierre Jobin, professeur de l'Université Laval et vice-président de la Société Richelieu, a rédigé un mémoire qui a été présenté à la Commission royale d'enquête sur l'avancement des arts, des lettres et des sciences (commission Massey), qui, tout en endossant plus ou moins la posture autonomiste de Duplessis, reconnaissait que le gouvernement fédéral avait un rôle à jouer dans le soutien de la production artistique et médiatique, lequel permettrait à chacune des « deux grandes races » du Canada de se développer selon sa "personnalité propre ${ }^{24}$ ». Jobin liait aussi le destin du Québec au sort des minorités canadiennes-françaises et distinguait la culture globale

20 "L'épouse d'un Richelieu... la vie du Richelieu ", Le Richelieu, vol. 11, n ${ }^{\text {os }}$ 5-6 (septembre-octobre 1961), p. 2, CRCCF, Fonds du Club Richelieu Ottawa (ciaprès Fonds CRO), C117-2/10/17.

${ }^{21}$ Émile Boucher, «Société Richelieu », Relations, décembre 1948, p. 160.

22 "Procès-verbal de la quatrième assemblée annuelle ", 20 février 1954, p. 9, CRCCF, Fonds RI, C76/1/4; « Éclatant succès : plus de 400 visiteurs présents à ces assises annuelles ", La Frontière, 21 septembre 1954.

23 "Congrès des Richelieu », 19 septembre 1949, CRCCF, Fonds CRO, C117-1/9/1.

${ }^{24}$ Pierre Jobin, Mémoire à la Commission royale d'enquête sur l'avancement des arts, des lettres et des sciences au Canada, Ottawa, Société Richelieu, janvier 1950, p. 6-7. 
(la nation) de la communauté politique (l'État fédéral partagé), dont faisaient partie les Canadiens français ${ }^{25}$.

À l'extérieur de la " ceinture bilingue ${ }^{26}$ " entourant le Québecsoit l'Est et le Nord-Est ontariens, la moitié septentrionale du Nouveau-Brunswick, ainsi que certains milieux de la NouvelleAngleterre -, les clubs de Windsor (Ontario) et de Saint-Boniface vivotaient. D'ailleurs, le siège social s'abstenait de fonder des cercles dans les milieux éloignés ou peu favorables au fait français, car il était difficile de les rejoindre (faute de moyens) et de susciter une entraide entre les clubs éloignés les uns des autres. Donc, le Richelieu réussissait surtout à renforcer les liens entre les îlots les plus rapprochés du Québec, en tenant de grands congrès pour que des réseaux et des amitiés émergent organiquement ${ }^{27}$. Lorsqu'on tenait un congrès annuel à l'extérieur de la vallée laurentienne (environ un congrès sur deux), on profitait du passage à Windsor (1957) ou à Moncton (Nouveau-Brunswick, 1958) pour promouvoir l'histoire et les exploits locaux des Canadiens français ou des Acadiens ${ }^{28}$. On a dû recevoir ces idées de manière très variable selon l'expérience et la connaissance des membres, mais la probabilité d'éveiller un sentiment de solidarité et de susciter des amitiés n'était pas négligeable. Un délégué du Québec a résumé ainsi ses impressions au Devoir à l'issue du congrès de 1957 :

J'y ai rencontré un groupe extrêmement sympathique, avide d'entendre des conférenciers du Québec et au sein duquel évo-

25 Lettre de Pierre Jobin à Horace Racine, 27 mars 1950, CRCCF, Fonds CRO, C117-1/9/5.

${ }^{26}$ Richard Joy, Languages in Conflict: The Canadian Experience, Toronto, McClelland and Stewart, 1972.

27 "Procès-verbal de la onzième assemblée [...] du Conseil d'administration ", 29 mai 1954; "Procès-verbal de la vingt-sixième assemblée [...] ", 23 janvier 1958, CRCCF, Fonds RI, C76/1/4 et C76/1/6.

28 "Oye Richelieu!", Bulletin du Club Richelieu Windsor, Ont., août 1956, Archives privées du Club Richelieu Manchester (ci-après APCRM), vol. «1956 »; " Procèsverbal de la vingt-septième assemblée [...] ", 15 décembre 1956, p. 2, CRCCF, Fonds RI, C76/1/5. 
luaient des personnes ayant conquis les épaulettes de la notoriété parmi la population de Windsor... Dans un Ontario où les clubs sociaux font florès, à deux pas des États-Unis, où il existe également un engouement pour ces institutions, les clubs Richelieu se révèlent comme une formule rajeunie et adéquate de propagande et de liaison qui ne peut que servir parfaitement les vues et les ambitions légitimes des Canadiens français... Il ne cherche évidemment pas à [se] substituer à la paroisse et à l'école, mais rejoignant la mentalité anglaise sur son propre terrain, il est plus apte ainsi à la tenir en respect ${ }^{29}$.

Plusieurs membres faisaient d'ailleurs la route jusqu'à Campbellton (Nouveau-Brunswick) ou Manchester (New Hampshire) pour assister aux remises de charte où ils saluaient leurs " fiers " confrères "dont l'esprit se nourri[ssai]t aux mêmes sources que le [leur] "30", preuve encore que la rhétorique nationale du mouvement minimisait souvent les différences entre les membres de différentes localités pour souligner les défis communs à la survivance française en Amérique.

Par ailleurs, cette sensibilisation aux enjeux en milieu minoritaire se faisait aussi au Québec. Le cercle de Montréal, par exemple, a tenu plusieurs conférences à ce sujet, auxquelles il invitait des membres des milieux minoritaires. S'il a critiqué, à l'occasion, la mainmise de l'élite d'Ottawa sur la direction du mouvement ${ }^{31}$, le cercle de la métropole dépensait plus d'énergie à déplorer les « difficultés politiques [...] des minorités nationales ${ }^{32}$ » ainsi que les injustices que subissaient les jeunes "francos » dans le domaine scolaire ${ }^{33}$. Les exposés, souvent présentés devant des notables, dont les historiens Guy Frégault et

${ }^{29}$ Le Richelieu, vol. 8, no 4 (juillet-août 1958), p. 11-12, CRCCF, Fonds CRO, C117-2/10/17.

30 Acadiana, 17 mars 1950, Bibliothèque et Archives nationales du Québec, Montréal (ci-après BAnQ-M), Fonds Club Richelieu Montréal (ci-après Fonds CRM), P206/8/91.

31 "Réunion mensuelle du Conseil d'administration ", 21 janvier 1946; "Procèsverbal de l'assemblée spéciale au bureau d'administration ", 15 août 1947, p. 70, APRI, vol. " Procès-verbaux 1944-1949".

32 Bulletin, 16 août 1948, BAnQ-M, Fonds CRM, P206/8/88.

33 Bulletin, 10 mai 1954, p. 3, BAnQ-M, Fonds CRM, P206/9/94. 
Lionel Groulx, ont parfois abouti à un don ponctuel pour une cause ${ }^{34}$. C'est ainsi qu'à la suite du troisième Congrès de la langue française à Québec en 1952, le cercle de Montréal a prélevé 15000 \$ pour les œuvres du Conseil de la vie française en Amérique. Au moment des célébrations du bicentenaire du Grand Dérangement, ce même cercle a aussi écouté l'auteur Calixte Savoie parler du passage de l'idéologie de la survivance à l'ambition nationale que l'Acadie s'apprêtait à franchir ${ }^{35}$. Le cercle de Manchester (New Hampshire), le premier aux États-Unis, est également parvenu à attirer des conférenciers de renom, dont le sénateur démocrate Jean-Charles Boucher ${ }^{36}$. Malgré le degré d'acculturation élevé des enfants franco-américains à l'époque, le poids démographique des générations précédentes maintiendrait une ambiance canadienne-française dans cette région pendant quelques décennies encore.

Puisque la diaspora canadienne-française constituait toujours un élément incontournable de la conscience nationale au Québec des années 1950, l'élite canadienne-française agissait presque par instinct lorsqu' elle tâchait de rassembler dans ses nouveaux clubs les descendants dispersés d'un empire français perdu. Bien entendu, il existait des divergences entre les cercles du Québec, qui vantaient surtout les mérites de la nation canadienne-française, ceux de l'Ontario et de l'Acadie, qui partageaient certaines postures de leurs confrères québécois tout en misant davantage sur la survivance et la bonne entente, et ceux de la Nouvelle-Angleterre, qui souhaitaient seulement ralentir l'hémorragie assimilationniste. Dans l'ensemble cependant, le poids de l'élite d'Ottawa au sein du conseil d'administration favorisait une conception canadienne-française de la nation, que

34 "Maillardville», 1953, p. 3, BAnQ-M, Fonds CRM, P206/9/94.

35 Calixte Savoie, "Les Acadiens sont à refaire leur Acadie ", La Patrie, 14 janvier 1955 , p. 2.

36 Lettre de Paul J. Gingras à Jean-Charles Boucher, 24 mai 1955; Lettre d'Adélard E. Côté à Paul J. Gingras, 9 mars 1956, APCRM, vol. «Assemblées préliminaires " et " Procès-verbaux et correspondance ", dossier «1955-1956". 
certains membres du Québec commençaient peut-être déjà à juger désuète.

Mais au-delà de cette solidarité nationale, germait un intérêt chez les membres pour l'étranger, l'immigration et les autres francophones de la planète. D'ailleurs, plusieurs Canadiens français avaient eu un contact direct avec les régions francophones de l'Europe pendant la Seconde Guerre mondiale ${ }^{37}$. Par ailleurs, en 1956, la Commission royale d'enquête sur les problèmes constitutionnels (commission Tremblay) publiait son rapport et proposait simultanément, peut-être un peu paradoxalement, que le Québec abandonne les souscriptions destinées au développement des collectivités minoritaires canadiennesfrançaises, une pratique jugée analogue aux compétences provinciales, mais qu'il développe des programmes dans ces mêmes champs avec la France ${ }^{38}$. L'année suivante le Richelieu a entrouvert la porte du recrutement aux parlants français qui n'étaient pas d'origine canadienne-française ou acadienne ${ }^{39}$. Les clubs du Québec avaient aussi commencé, vers 1950, à préconiser l'acculturation des immigrants catholiques à la société canadienne-française. Plus timidement, le cercle d'Ottawa souhaitait plutôt « que [son] groupe ethnique puisse [aussi] profiter de la venue de ces nouveaux collaborateurs à la vie canadienne ${ }^{40}$ ". Les cercles de Montréal et d'Ottawa ainsi que celui de Manchester se sont penchés sur les enjeux diplomatiques en général, mais ont surtout multiplié les rencontres avec les ambassadeurs et les

37 Sean Mills, Contester l'empire : pensée postcoloniale et militantisme politique à Montréal, 1963-1972, traduit de l'anglais par Hélène Paré, Montréal, Hurtubise HMH, 2011; David Meren, "An Atmosphere of Libération: The Role of Decolonization in the France-Quebec: Rapprochement of the 1960s ", The Canadian Historical Review, vol. 92, n 2 (juin 2011), p. 263-294.

38 Robin S. Gendron, Towards a Francophone Community: Canada's Relations with France and French Africa, 1945-1968, Montréal, McGill-Queen's University Press, 2006.

39 "Procès-verbal de la sixième assemblée régulière du Conseil d'administration ", 13 avril 1957, p. 3, CRCCF, Fonds RI, C76/1/6.

40 "Dîner hebdomadaire ", 13 juin 1951, p. 3, CRCCF, Fonds CRO, C117-1/9/1. 
diplomates français et haïtiens pendant ces années ${ }^{41}$. Dès 1952, on a tenu quelques discussions à Montréal et à Ottawa sur le rapprochement des peuples canadien-français et français afin de mieux résister au rouleau compresseur culturel anglo-américain ${ }^{42}$. L'idée a même atteint le cercle de Timmins (Ontario), qui a suggéré en 1953 qu’on fonde bientôt des cercles en France et en Afrique française ${ }^{43}$. Ce souhait s'exprimait dans le contexte où, entre 1948 et 1958, les Lions Clubs s'établissaient en France, au Luxembourg, à Saint-Pierre-etMiquelon, ainsi qu'au Burkina Faso, au Gabon et au Niger ${ }^{44}$. Dès 1946, le Richelieu a envisagé la possibilité d'établir un cercle à Portau-Prince (Haïti), puisqu'on estimait partager la même langue (on occultait la créolisation) et la même foi avec les Haïtiens, mais la proposition n'a pas mené à des efforts formels pendant ces années ${ }^{45}$.

En somme, devant les préoccupations distinctes du Québec et des collectivités canadiennes-françaises en milieu minoritaire, le Richelieu a privilégié les réalités communes, soit les succès et les défis qui rejoignaient l'ensemble des parlants français du continent pendant la période. Les minorités des milieux partagés avec la majorité anglophone, en disposant de moins d'institutions au service de leur collectivité et d'un plus faible rapport de force pour leur langue dans l'espace public, vivaient évidemment cette marginalisation de manière plus quotidienne. Cela dit, le Richelieu a surtout encouragé ses clubs à arrimer les réalités particulières de leurs localités aux problématiques

41 Bulletin, 13 septembre 1954, BAnQ-M, Fonds CRM, P206, bobine 6391, image 1599; "Dîner hebdomadaire », 11 avril 1951, p. 5, CRCCF, Fonds CRO, C117-1/9/1; Lettre de Paul J. Gingras à Charles de Pampelonne, 20 avril 1956, APCRM, vol. " Procès-verbaux et correspondance ", dossier "1955-1956 ".

42 "La France victorieuse et glorieuse sous ses rois reprendra un jour son rôle ", La Patrie, 11 novembre 1953.

43 "Vie des clubs, vue de loin", Le Richelieu, vol. 3, n 1 (février 1953), p. 4, BAC, Collection d'ouvrages, J.257.2.

44 Paul Martin, We Serve: A History of the Lions Clubs, Washington, Regnery Gateway Publishing, 1991, p. 255, 302-305.

45 Lettre de Carlet Auguste à Paul J. Gingras, 21 août 1959; Lettre de Paul J. Gingras à Carlet Auguste, 23 novembre 1959, APCRM, vol. « 1959 »; Bulletin, 20 mai 1955, BAnQ-M, Fonds CRM, P206, bobine 6392, image 98. 
communes à l'ensemble des parlants français. Étant donné qu'un peuple devait posséder des « raisons communes " pour se reconnaître et s'imaginer un avenir collectif, aurait dit Fernand Dumont, le Richelieu a suscité une solidarité nouvelle en affiliant des cercles de l'Acadie aux Prairies et du Saguenay au Rhode Island, d'autant plus qu'il ne constatait aucune contradiction, à l'époque, entre l'autonomisation du Québec, le soutien à la diaspora et le développement de rapports embryonnaires avec les autres peuples francophones.

\section{Une nouvelle rivalité entre divers projets francophones (1960-1972)}

Plusieurs acteurs de la décennie 1960 soulignent la rapidité et la profondeur des ruptures sociales, culturelles et politiques, mais les historiens et sociologues, dont Michael Gauvreau, E.-Martin Meunier et Jean-Philippe Warren, insistent aujourd'hui sur les signes avant-coureurs - notamment le renouveau catholique et la prospérité de l'après-guerre - qui auraient annoncé les turbulences à venir ${ }^{46}$. La dynamique créée par l'évolution particulière du Canada français et l'influence des courants sociaux et idéologiques occidentaux en son sein, soit l'explosion démographique du baby-boom, la prospérité économique, la multiplication des programmes sociaux, la démocratisation de l'éducation ainsi qu'un catholicisme axé sur l'engagement du croyant dans son milieu ont tous contribué à miner le projet national paraétatique canadien-français. En revanche, si on a étudié le rôle accru de l'État comme mécanisme redéfinissant les identités, ainsi que la mondialisation des référents culturels, on n'a à peu près rien dit sur l'influence du mouvement de la Francophonie et son corrollaire, l'identité « francophone », sur la fragmentation idéologique et institutionnelle du Canada français. Le politologue Simon

46 Michael Gauvreau, Les origines catholiques de la Révolution tranquille, traduit de l'anglais par Richard Dubois, Montréal, Éditions Fides, 2008; E.-Martin Meunier et Jean-Philippe Warren, Sortir de la grande noirceur : l'horizon personnaliste de la Révolution tranquille, Sillery, Éditions du Septentrion, 2002. 
Langlois a montré l'existence d'un lien entre la fragmentation du Canada français et la mondialisation des référents identitaires - dans la mesure où la prolifération des droits individuels, des déplacements et des communications à l'échelle planétaire aurait mené à l'émergence des références supranationales -, sans toutefois référer explicitement à la Francophonie ${ }^{47}$. Cette " ambiance de libération " dont parle David Meren a aussi eu un effet sur la fragmentation du Canada français et sur son intégration à un nouvel ensemble mondial. Cette mutation n'a pas laissé le Richelieu indemne, tant sur le plan idéologique que sur le plan institutionnel...

Certes, c'est la marche vers la modernisation et l'autodétermination qui animait les Canadiens français pendant la Révolution tranquille qui retient l'attention de prime abord. Les possibilités de changer le rapport au politique dans le cadre d'un fédéralisme renouvelé ou d'un Québec indépendant ont toutefois suscité différentes résistances qui existent toujours tant du côté anglophone que francophone. Dans ce processus d'affirmation de l'État québécois, conçu comme un mécanisme de développement collectif, le réseau institutionnel catholique et canadien-français a joué un grand rôle, mais il a tout de même fini par perdre beaucoup d'importance aux yeux des Québécois. De leur côté, les minorités canadiennes-françaises et acadienne étaient également emballées, mais dans une moindre mesure, par les possibilités qu'offraient leurs provinces respectives pour leur avancement social et culturel, même si elles auraient probablement préféré ménager les réseaux associatifs tout en profitant des nouvelles possibilités sociopolitiques. L'évacuation de l'idéologie canadienne-française au Québec pendant la décennie de même que l'émergence du mouvement indépendantiste au sein des forces nationalistes ont fini par mettre fin à l'Ordre de Jacques-Cartier en 1965 et précipité le départ de certaines délégations minoritaires des

47 Simon Langlois, «Un cas typique de mutation de la référence nationale : le Canada français ", dans Simon Langlois (dir.), Identités et cultures nationales : l'Amérique française en mutation, Québec, Les Presses de l'Université Laval, 1995, p. 3-14. 
États généraux du Canada français deux ans plus tard. Il s'agissait d'un second fossé qui se creusait, qui n’allait pas bientôt être oublié chez les minorités...

Malgré ces remaniements institutionnels et idéologiques, la nation canadienne-française est demeurée une référence importante au cours de la décennie 1960, comme le révèlent les archives du Richelieu, même si les références explicites à une cause ou à une mémoire commune ont commencé à se faire plus rares. Par exemple, le mouvement a incité l'État fédéral à développer les services radiophoniques et télévisuels de Radio-Canada et à adopter des symboles canadiens en 1963 et $1964^{48}$. Dans le cadre des consultations de la Commission royale d'enquête sur le bilinguisme et le biculturalisme (commission Laurendeau-Dunton), le cercle de Montréal a recommandé l'établissement de districts bilingues pour que les deux langues majoritaires soient employées dans l'espace public et commercial et "pour que se concrétise dans les faits la réalité biculturelle ${ }^{49}$ ". Toutefois, preuve des tensions que suscitait la question nationale à ce moment-là, le Richelieu n’a déposé aucun mémoire à la commission Laurendeau-Dunton, même s'il n'avait pas hésité à en présenter un à la commission Massey en 1950. En suivant de près l'effondrement de l'Ordre de Jacques-Cartier, il semble avoir préféré ne pas s'avancer sur un terrain qui pouvait le mettre dans l'embarras. Il a cependant continué à tenir la moitié de ses congrès annuels en périphérie du Québec, dont à Manchester en 1960, où 1600 délégués ont reconnu le « mérite de leurs compatriotes ${ }^{50} »$. On estimait vouloir y maintenir un certain discours axé sur la survivance, que le Québec soit de la

48 "Procès-verbal de la douzième réunion du Conseil d'administration ", 18 juillet 1963 ; " Procès-verbal de la huitième réunion du Conseil d'administration ", 11 juin 1964, CRCCF, Fonds RI, C76/1/8 et C76/2/1.

49 Club Richelieu-Montréal et Rotary Club of Montreal, "Mémoire conjoint à la Commission royale d'enquête sur le bilinguisme et le biculturalisme ", décembre 1964, p. 11-12, BAC, Collection d'ouvrages, CA1 2163 B31 B209.

50 "Procès-verbal de la vingt-quatrième assemblée régulière du Conseil d'administration ", 17 janvier 1963, p. 2; «Rapport de la réunion du comité des congrès ", 27 février 1964, CRCCF, Fonds RI, C76/1/8 et C76/2/1. 
partie ou non ${ }^{51}$. Si ces grand-messes attiraient toujours des politiciens, dont l'ancien premier ministre du Canada Louis Saint-Laurent ${ }^{52}$, un doute s'installait déjà, entre autres, au club de Québec, concernant l'avenir d'une "poignée de Français [...] perdus dans la masse ${ }^{53}$ " anglo-saxonne.

Le Richelieu n'a pu se soustraire entièrement aux déchirements sur l'avenir du Canada français. Par exemple, lorsque certains membres hullois du cercle d'Ottawa-Hull avaient soulevé l'idée, en 1946 et en 1956, de former un cercle autonome sur la rive québécoise, le conseil d'administration avait réussi à écarter l'idée, en rappelant que ce cercle symbolisait la pérennité de la solidarité outre-frontières ${ }^{54}$. Il a toutefois fallu plus de doigté, à l'automne 1962, pour réagir à la formation d'un cercle clandestin à Hull, que ses instigateurs présentaient comme un meilleur moyen de recruter des Canadiens français "embrigadés ${ }^{55}$ » dans les clubs neutres. Les sections locales de l'Ordre de Jacques-Cartier et de la Société Saint-Jean-Baptiste sont intervenues et ont exercé des pressions sur l'élite d'Ottawa pour qu'elle reconnaisse, enfin, les aspirations des requérants hullois ${ }^{56}$. "C'était une division dans la maison canadienne-française ${ }^{57}$ ", rappelle aujourd'hui le membre ottavien Laurent Isabelle. Le départ des membres de Hull

${ }^{51}$ Lettre de Normand-W. Lachance à Adolphe Robert, 14 mai 1960; «Three-Day Convention Attracts 1600 Visitors ", Manchester Union Leader, 17 septembre 1960, APCRM, vol. " [Sans titre] » et «1960-1961".

52 Bulletin, 27 septembre 1960, BAnQ-M, Fonds CRM, P206, bobine 6392, image 645; Le Richelieu, $\mathrm{n}^{\circ}$ 10, numéro spécial, 1960, p. 2, CRCCF, Fonds CRO, C117-2/10/17.

53 Bulletin, 18 avril 1960, p. 1, APCRQ, vol. «Bulletins ", dossier « 1960 ».

54 "Procès-verbal de la dix-neuvième assemblée régulière du Conseil d'administration ", 13 septembre 1956, CRCCF, Fonds RI C76/1/5.

55 Lettre de Suzanne Gosselin au gouverneur, 14 février 1963, APRI, vol. « Clubs », dossier « 07126 Hull ».

56 "Rapport de la réu[n]ion du C.P.R. », 29 octobre 1962; " Lettre du secrétaire régional au secrétaire ", 18 décembre 1962, CRCCF, Fonds OJC, C3/29/6; "Procès-verbal de la dixième assemblée régulière du Conseil d'administration ", 26 juillet 1962, CRCCF, Fonds RI, C76/1/5.

57 Entrevue avec Laurent Isabelle, Ottawa (Ontario), 21 juin 2011. 
a laissé une certaine amertume chez les membres franco-ontariens, qui ont maintenu le nom de leur club - «Ottawa-Hull» - pendant trois ans et conservé le capital amassé sur les deux rives dans son fonds des œuvres ${ }^{58}$. En 1965, le cercle du Témiscamingue s'est également divisé en sections québécoise et ontarienne, peut-être pour des raisons semblables.

La division des deux cercles chevauchant la frontière du Québec aurait pu s'inspirer de la division des diocèses de Haileybury et d'Ottawa pendant les années 1960, mais elle semble surtout avoir eu lieu en raison des oppositions sur la question nationale qui parvenaient déjà à ébranler des amitiés. Il se peut bien que le Richelieu ait tenté de calmer ces tensions en s'abstenant de participer aux États généraux, un geste qui a d'ailleurs bien déçu son organisateur Rosaire Morin ${ }^{59}$. Certes, plusieurs membres y participaient déjà par l'entremise d'autres organismes, mais le conseil d'administration avouerait plus tard qu'il avait été sage de s'en tenir loin, vu le départ fracassant des porteparoles franco-ontariens et acadiens pendant les assises de novembre $1967^{60}$. Pour sa part, le Richelieu s'est contenté de recevoir quelques subventions, dont $4000 \$$ pour le congrès de Toronto en $1966^{61}$, du Service du Canada français d'outre-frontières (SCFOF), fondé quelques années plus tôt pour remplacer le soutien privé au réseau institutionnel des minorités canadiennes-françaises et acadienne.

Bien entendu, le Richelieu avait un penchant plus social et intellectuel que politique, surtout si on le compare à l'Ordre de Jacques-Cartier. Il ne menait pas directement d'interventions dans

58 Bulletin, 25 juin 1963, p. 2 ; E. J. Whiteside, «Re : Club Richelieu Ottawa-Hull change of name to Club Richelieu Ottawa », 8 juin 1965, CRCCF, Fonds CRO, C117-2/5/8 et C117-2/7/20.

59 "Procès-verbal de la première réunion du Conseil d'administration ", 12 mars 1964, p. 2, CRCCF, Fonds RI, C76/2/1.

60 "Procès-verbal d'une réunion du Comité de Régie ", $1^{\text {er }}$ juin 1967; Jean-Jacques Tremblay, "Les États généraux », [1968], CRCCF, Fonds RI, C76/2/4, et Fonds Jean-Jacques Tremblay (ci-après Fonds JJT), P195/1/10.

${ }^{61}$ "Québec, merci!", Le Richelieu, vol. 16, nº 3 (mai-juin 1966), p. 16, APRI, vol. «Vie Richelieu 1946-1980 ». 
la sphère politique pour rehausser la place des Canadiens français, mais privilégiait surtout des œuvres caritatives pour leurs jeunes compatriotes. S'il n'a pas pris position sur l'autonomie du Québec à l'inverse de l'OJC et de la SSJB -, le Richelieu a toutefois été marqué par la volonté des cercles québécois d'acquérir plus d'autonomie par rapport au siège social et l'élite d'Ottawa. C'est ainsi qu'il a inauguré, dès 1964, des conseils régionaux, qui coordonneraient localement des projets caritatifs et culturels pour les cercles d'une région. Preuve encore que la décentralisation de l'organisme semblait presque inévitable, en février 1968, il a aussi mis sur pied douze districts, dont les membres éliraient un représentant au conseil d'administration. Par contre, l'élite d'Ottawa a tout de même profité de sa marge de manœuvre pour assurer que le Richelieu créé des districts régionaux qui ne suivaient pas les frontières politiques. Les clubs ont donc été regroupés dans les régions (Nord, Outaouais et Baie des Chaleurs, par exemple) au lieu de suivre les frontières provinciales.

Cette mesure constitue une des raisons pour lesquelles le Richelieu a évité la québécisation et la disparition. Sean Mills et Joel Belliveau rappellent d'ailleurs que le libéralisme social de l'époque, véhiculé par les divers spectacles de contre-culture chez les jeunes au Québec et en Acadie, a fini par marquer plusieurs adultes de manière considérable dès la fin de la décennie $1960^{62}$. Malgré son traditionalisme, le Richelieu a pris la balle au bond pour s'adapter, du moins en partie, aux sables mouvants des mutations sociales et politiques de l'époque. Il a tenté de maintenir une assise canadienne-française tout en reconnaissant la volonté d'autonomisation de ses cercles québécois. Si ces derniers semblaient s'éloigner des îlots minoritaires, qui dérivaient peut-être même à perte de vue chez certains, le Richelieu a diminué la fréquence et l'intensité de sa rhétorique sur les réalités partagées entre le Québec et les minorités, juste assez pour qu’il n'apparaisse pas comme étant analogue aux nouvelles aspirations de

62 Joel Belliveau, Le " moment 68 " et la réinvention de l'Acadie, Ottawa, Les Presses de l'Université d'Ottawa, 2014 ; Mills, Contester l'empire. 
cette majorité, mais pas trop pour que le Richelieu n'apparaisse plus en phase avec les aspirations des minorités, ainsi que le " respect " qu'elles maintenaient toujours pour le projet national d'antan.

On ne doit pas y voir un lent abandon du " rêve d'un pays imaginé ", mais plutôt le passage d'une rhétorique explicite à un comportement implicite. À partir de 1967, on ne vantait plus beaucoup les mérites du projet national canadien-français, mais lorsque venait le temps d'élire la présidence par exemple, les membres maintenaient une alternance presque parfaite entre un membre du Québec et un membre de sa périphérie. Au printemps 1968, le mouvement a aussi tâché d'étouffer dans l'œuf la volonté d'au moins deux cercles, ceux de La Pocatière et de Longueuil, de travailler ouvertement pour l'indépendance du Québec. Ceux-ci s'étaient laissé " exploit[er] [...] par les partis politiques ou groupes indépendantistes ${ }^{63}$ " et devaient faire preuve d'un " esprit ouvert [...] sur la collectivité nationale ${ }^{64} "$, selon les administrateurs à Ottawa. En revanche, cette volonté de ménager la chèvre et le chou semble avoir encouragé le départ des franges les plus nationalistes de l'effectif du Québec et découragé l'engagement de bénévoles vis-à-vis un mouvement qui refusait de se positionner sur l'indépendance. Les sources ne sont pas explicites à cet égard, mais on voit difficilement comment l'un des cercles les plus nationalistes et actifs pendant une vingtaine d'années, celui de Montréal, serait passé de 175 à 30 membres en quelques années ${ }^{65}$. Par ailleurs, deux autres projets d'affiliation sur l'île ont échoué à la fin de la décennie, semble-t-il, en raison des fortes allégeances politiques parmi les recrues ${ }^{66}$. Quelques clubs québécois

63 "Procès-verbal de la deuxième réunion du comité de la coordination ", 14 juin 1968, p. 3, CRCCF, Fonds RI, C76/2/6.

64 "Procès-verbal du comité de régie ", 5 avril 1968, p. 2, CRCCF, Fonds RI, C76/2/5.

65 "Rapport d'activités de l'agent de liaison ", 6 octobre 1969, p. 2 ; « Procès-verbal de la réunion des comités d'expansion et de fondations [sic]», 12 juin 1970, CRCCF, Fonds RI, C76/2/6 et C76/2/7.

${ }^{66}$ " Procès-verbal de la neuvième réunion du Conseil d'administration ", 22-23 janvier 1971, p. 2, CRCCF, Fonds RI C76/2/7. 
ont aussi fermé leurs portes. Le mouvement semble donc avoir stagné, en partie du moins, à cause de ce refus d'adopter une position claire sur la question nationale ${ }^{67}$.

La période entre 1968 et 1972 apparait, en contraste, tout autrement dans les milieux francophones à l'extérieur de la Belle Province, où l'engouement pour les mouvements associatifs est demeuré important. Devant la disparition de certains regroupements catholiques, dont l'Action catholique, et nationaux, dont les sections de la SSJB, le Richelieu a pu actualiser sa pertinence sociale et culturelle et y recruter d'anciens membres. Le mouvement a même intensifié le rythme des fondations en Ontario français et en Acadie au tournant des années 1970. Les nouvelles banlieues d'Ottawa (Orléans) et de Toronto (Oshawa) ont constitué de nouveaux cercles, tandis que d'autres cercles continuaient de promouvoir le pacte entre deux peuples ${ }^{68}$, peut-être afin de mieux distinguer les FrancoOntariens des autres groupes ethniques de la province. Pour sa part, le cercle d'Ottawa a continué de pousser la réflexion publique sur l'ouverture d'écoles secondaires de langue française et n'hésitait pas à encourager les élèves à faire pression sur les commissions scolaires publiques récalcitrantes ${ }^{69}$. En Nouvelle-Angleterre francophone, la lutte pour les droits civiques, associée aux effets de la Révolution tranquille, a maintenu l'engagement des membres et attiré de nouveaux chefs de file franco-américains au Richelieu. Devant l'effondrement des écoles paroissiales bilingues et de certaines institutions franco-américaines, ceux-ci ont tenté de tailler une place au français

67 Diane Vallée, Cinquantenaire du Club Richelieu Mont-Joli : rappels et reconnaissance, 1946-1996: célébration, 8 décembre 1996, Mont-Joli, Club Richelieu de MontJoli, 1996, [annexe 1].

68 "Procès-verbal de la réunion des comités d'expansion et de fondations [sic] ", 12 juin 1970, CRCCF, Fonds RI, C76/2/6 et C76/2/7; La Revue Richelieu (mai-juin 1969), p. 59.

69 "An Act to amend The Secondary Schools and Boards of Education Act ", 1968; "Procès-verbal de la réunion du Conseil d'administration ", 20 septembre 1971, p. 2 ; Bulletin, 6 juillet 1972, p. 2, CRCCF, Fonds CRO, C117-1/7/10 et Fonds $\mathrm{CRO}, \mathrm{C} 117-2 / 5 / 12, \mathrm{C} 117-2 / 12 /$ et $\mathrm{C} 117-2 / 31$. 
dans les espaces de la majorité 70 . Au U.S. Department of Education ou au Sénat du New Hampshire, des membres ont demandé que l'on diffuse les ondes télévisuelles du Québec ou que l'on enrichisse les programmes de français dans les high schools publics. D'ailleurs, seul le cercle de Worcester (Massachussetts) a mis fin à ses activités en 1970, faute de relève. Les Cadiens de la Louisiane ont aussi participé au réveil en voulant y raviver la langue française. Grâce à une subvention de 20000 \$ du Service du Canada français d'outrefrontières, le Richelieu a réussi à affilier un premier club, composé d'enseignants de français, à Lafayette en $1969^{71}$.

La neutralité politique du Richelieu et sa volonté de s'adapter aux tendances sociales et politiques des " années 68 » ont donc parallèlement amené la stagnation du recrutement au Québec et une expansion ailleurs en Amérique française. Ce renouveau, avons-nous dit, mettait moins l'accent sur les particularités partagées entre les francophones du continent que sur les références et les identités régionales acadienne, franco-américaine, franco-ontarienne et québécoise.

Le Richelieu avait peut-être "évité de tomber dans la fosse de l'OJC ${ }^{72}$ ", se rappelle aujourd'hui l'ex-délégué Grégoire Pagé, mais le mouvement s'est aussi rendu vulnérable en se précipitant vers des tendances de l'heure, ce qui aurait des conséquences durables sur le mouvement. Alors que le gouvernement fédéral tentait de redéfinir le Canada comme le fleuron mondial du bilinguisme et du multicultu-

70 La Revue Richelieu, vol. 19, nº 2 (mars-avril 1969), p. 21, CRCCF, Fonds CRO, C117-2/10/19; "Procès-verbal d'une réunion du comité de régie ", 12 février 1970, p. 4, CRCCF, Fonds RI, C76/3/1 ; "French Language TV From Canada Coming When Obstacles Hurdled ", The Manchester Union-Leader, 16 septembre 1973, APCRM, vol. «1973».

71 "Procès-verbal de la réunion du comité de régie ", 25 octobre 1968; " Procèsverbal de la réunion du comité de régie », 25 janvier 1969, p. 3 ; "Procès-verbal de la cinquième réunion du Conseil d'administration ", 12 septembre 1969, p. 4 ; " Procès-verbal de la réunion du comité de régie ", 12 février 1970, p. 2; "Procès-verbal de la quatrième réunion du Conseil d'administration ", 13 juin 1970, p. 5, CRCCF, Fonds RI, C76/2/5, 6 et 7 et C76/1/3.

72 Entrevue avec Grégoire Pagé, Ottawa (Ontario), 10 mai 2011. 
ralisme, il portait un dur coup à la notion de dualité et à celle d'une fédération binationale. Récemment élu à la tête de son parti et devenu premier ministre, Pierre Elliott Trudeau a lui-même visité le club de Campbellton en mai 1968. Au cours de cette visite, il a avancé que la préservation de « la langue et $[\mathrm{d}]$ es traditions françaises » n'empêchait pas le relâchement des traits anachroniques d'un peuple. Il a même conclu en estimant que « les traditions [devaient] changer $^{73}$ ".

Pour atteindre cet objectif, le gouvernement fédéral de Trudeau ne s'est pas contenté de faire des déclarations auprès des citoyens. Le Secrétariat d'État a ainsi reçu le mandat de promouvoir la participation des citoyens à la vie publique, et celui d'offrir aux organismes en milieu minoritaire des subventions gouvernementales, toujours en vue de renouveler l'engagement vis-à-vis la fédération canadienne ${ }^{74}$. En offrant une subvention annuelle de 10000 \$ au Richelieu à partir de 1970, le Secrétariat d'État espérait encourager le développement des activités intellectuelles en milieu minoritaire ainsi que l'établissement de cercles dans l'Ouest canadien ${ }^{75}$. Ainsi, le Richelieu adopterait un caractère pancanadien et ne serait plus uniquement centré sur le Québec et sa périphérie. Par contre, lors de ses missions d'expansion, le délégué Paul-Émile Bélanger a vite été confronté à la quasiabsence du français dans plusieurs milieux professionnels urbains. Il a découvert non seulement une "mentalité $[. .$.$] très différente { }^{76}$ " chez les Canadiens français de Calgary, mais aussi une « attitude

73 Pierre Elliott Trudeau, dans La Revue Richelieu, mai-juin 1969, p. 62, BAC, Collection d'ouvrages, J257.2.

${ }^{74}$ Leslie Alexander Pal, Interests of State: The Politics of Language, Multiculturalism, and Feminism in Canada, Montréal, McGill-Queen's University Press, 1993, p. 101122; Michel Bock, "De la "tradition" à la "participation" : les années 1960 et les mouvements de jeunesse franco-ontariens ", Cahiers Charlevoix : études francoontariennes, vol. 8, Ottawa, Les Presses de l'Université d'Ottawa, 2010, p. 164.

75 Lettre de J. René Préfontaine à Paul Lumsden. «Société Richelieu Internationale », 16 avril 1970 ; Lettre de R. Paul Lumsden à René Préfontaine, 5 mai 1970, BAC, Fonds Secrétariat d'État, RG6-F-4, vol. CB9-213.

76 "Rapport de l'agent de liaison », 4 décembre 1970, p. 8, CRCCF, Fonds RI, C76/2/7. 
[négative] d'un fort pourcentage de l'élément anglophone envers les francophones ${ }^{77}$ » à Halifax, ce qui aurait découragé les professionnels acadiens en milieu urbain de s'affirmer dans leur langue. Malgré l'adoption récente de la Loi sur les langues officielles, au-delà des régions francophones et bilingues du Canada, les Canadiens français étaient toujours à peu près invisibles dans l'espace public. Le Secrétariat d'État a aussi proposé au Richelieu un "projet d'échange de jeunes d'expression française ${ }^{78}$ ", qui occultait les vocables identitaires, non seulement pour renforcer la connaissance des francophones hors Québec, mais aussi pour miner l'indépendantisme québécois. Devant "servir à l'unité nationale [canadienne] ${ }^{79}$ ", ce programme a souvent occulté les rapports de force politiques derrière la fragilité de plusieurs milieux pour réduire la vitalité d'un milieu à la simple volonté des individus. Sur le plan pragmatique toutefois, les clubs s'y engageaient simplement pour permettre à des jeunes de leurs localités de visiter le Canada ${ }^{80}$. Le succès de l'initiative a été tel que les anciens présidents ont même fini par suggérer au conseil d'administration d'" adapter la formule [du] Richelieu à l'idée de perspective jeunesse du gouvernement fédéral ${ }^{81} »$.

Outre l'intervention de l'État fédéral pour coopter, voire " canadianiser » le projet canadien-français, l'émergence d'une conscience francophone mondiale faisait aussi son apparition dans le paysage des mutations nationales. Certes, plusieurs missionnaires et religieuses de France avaient instruit ou soigné les Canadiens français, et la France demeurait un lieu d'inspiration culturelle ainsi qu'un

\footnotetext{
77 «Rapport de l'agent de liaison ", 8 février 1971, p. 5, CRCCF, Fonds RI, C76/3/6.

78 " Procès-verbal de la sixième réunion du Conseil d'administration », 29 novembre 1968, CRCCF, Fonds RI, C76/2/5.

79 «Rapport des délibérations entendues lors d'une réunion du Comité d'échanges culturels », 27 mars 1969, CRCCF, Fonds RI C76/2/6.

80 "Procès-verbal de la troisième réunion du Conseil d'administration ", 28 avril 1972, p. 6; " Procès-verbal de la quatrième réunion du Conseil d'administration ", 9 juin 1972, CRCCF, Fonds RI, C76/3/3.

81 "Rapport de la réunion du comité des présidents généraux ", 22-23 octobre 1971, p. 2, CRCCF, Fonds RI, C76/3/3.
} 
partenaire économique et un allié militaire du Canada, mais les liens entre les sociétés civiles canadienne et française demeuraient rares ${ }^{82}$. Le développement des transports et des communications de même que l'émergence des ambitions internationales du Québec et des autres peuples francophones ont contribué à l'apparition d'une conscience des espaces francophones du monde ${ }^{83}$. Pendant que le réseau institutionnel canadien-français s'effritait, des nationalistes au Québec ont jeté les bases d'une nouvelle solidarité outre-mer en fondant l'Union des journalistes de langue française en 1954 et l'Association des universités partiellement ou entièrement de langue française (AUPELF) en 1961. Le Richelieu a songé, dès 1965, à la possibilité d'affilier des cercles en France avec l'ambassadeur de France au Canada, François Leduc. Par ailleurs, le mouvement a tenu son premier congrès annuel outre-mer à Cannes (France) en septembre 1968. Au niveau local, les cercles s'intéressaient de plus en plus à l'Europe et à la décolonisation des pays du Sud, souvent en invitant à leur tribune un ambassadeur ou un missionnaire venant du pays en question ${ }^{84}$.

Devant trois mutations culturelles - l'autonomisation du Québec, l'édification d'un nouveau nationalisme canadien et la mondialisation de la Francophonie -, le Richelieu a trouvé une échappatoire à la crise du Canada français en se tournant vers la Francophonie mondiale. Le président du mouvement en 1968-1969 avait beau encore être nationaliste canadien-français au sens traditionnel du terme, Gontran

${ }^{82}$ Robert Bothwell, Alliance and Illusion: Canada and the World, 1945-1984, Vancouver, University of British Columbia Press, 2007, p. 300 ; David Meren, With Friends Like These: Entangled Nationalisms and the Canada-Quebec-France Triangle, 1944-1970, Vancouver, University of British Columbia Press, 2012.

${ }^{83}$ Serge Berstein, Histoire du gaullisme, Paris, Les Éditions Perrin, 2001, p. 514.

84 "Conférence ", Bulletin, 28 février 1965, APCRM, vol. " 1965 »; Lettre de Dominique Agoumba au Club Richelieu à Montréal, 15 septembre 1960, BAnQ-M, Fonds CRM, P206, bobine 6392, image 667; "Procès-verbal de la quinzième assemblée générale annuelle ", 19-20 février 1965, p. 2; Le Richelieu, vol. 15, no 6 (novembre-décembre 1965), p. 2, CRCCF, Fonds RI, C76/2/1 et Fonds CRO, C117-2/1 / et C117-2/17. 
Rouleau est venu à croire qu'il y avait peut-être moyen d'empêcher la disparition du projet canadien-français - et celle du Richelieu - en mobilisant les francophones d'outre-mer. Autrement dit, on parviendrait peut-être à concilier les liens de solidarité avec la diaspora et les ambitions internationales du Québec en érigeant un nouvel échelon, au-delà des projets régionaux, fédéral et national ${ }^{85}$. Né à Matane (Québec) en 1927 et ayant étudié le droit à l'Université de Montréal, Rouleau a par la suite déménagé à Baie-Comeau ${ }^{86}$. Il s'est engagé au sein du club local dans la vingtaine et est devenu l'un des plus jeunes présidents du mouvement à l'âge de trente-deux ans. Nationaliste, mais aussi fédéraliste et trahissant parfois son allégeance au Parti libéral, il souhaitait éviter que le mouvement devienne une autre victime de la crise nationale ${ }^{87}$. La mondialisation du mouvement pouvait ainsi représenter une "planche de salut " pour le Richelieu, menacé de disparition. Elle proposait, écrivait-il, un «traitement [de] choc nécessaire pour assurer la conservation et la consolidation à l'intérieur des cadres traditionnels [... des relations interprovinciales ${ }^{88}$ ". "Français, [...] Franco-Ontariens, Acadiens, Franco-Américains et Canadiens français du Québec » pourraient ainsi "dialoguer et fraterniser ${ }^{89}$ " entre eux et assurer que le Québec conserve des liens avec sa diaspora. Selon Rouleau, le Québec avait beau se tourner vers la France, quoi qu'il fasse sur les plans politique et culturel, il resterait tout de même « indissolublement lié au continent nord-américain ${ }^{90}$ "

${ }^{85}$ Gontran Rouleau, "Le mythe-équation : langue et foi ", Vie Richelieu, vol. 18, dossier 5 (septembre 1968), p. 13, CRCCF, Fonds CRO, C117-2/10/19.

${ }^{86}$ Jean Cournoyer, "Rouleau (Gontran) ", La Mémoire du Québec, [En ligne], [http://www.memoireduquebec.com/wiki/index.php?title=Rouleau_(Gontran)] (28 mai 2015).

87 Gontran Rouleau, «À l'issue d'un tour de France : conférence de presse à Paris en marge de l'expansion Richelieu ", La Revue Richelieu, mai-juin 1969, p. 46.

${ }^{88}$ Gontran Rouleau, La Revue Richelieu, vol. 24, no 4 (juillet-août 1974), p. 12, CRCCF, Fonds CRO, C117-2/10/19.

89 Gontran Rouleau, «Déjeuner Richelieu à Cannes ", La Revue Richelieu, vol. 18, $n^{\circ} 6$ (novembre 1968), p. 3, CRCCF, Fonds CRO, C117-2/10/19.

90 Gontran Rouleau, "À l'issue d'un tour de France », p. 46. 
sur le plan géographique. Par conséquent, le Québec n'avait d'autre choix que de se préoccuper du sort des avant-postes francophones ailleurs sur le continent ${ }^{91}$.

Jusque-là, les promoteurs de la Francophonie mondiale avaient surtout été des littéraires à l'extérieur de l'Hexagone, dont le Québécois Jean-Marc Léger, le Sénégalais Léopold Senghor et le Tunisien Habib Bourguiba. Pendant la décennie 1960, leurs efforts auraient fait germer une conscience francophone dans les nouveaux États, issus de la décolonisation française, et dans les espaces où la langue française occupait une place importante ${ }^{92}$. Comme la France ne possédait plus l'autorité morale de mener la Francophonie sans être accusée de néocolonialisme, ses interventions se sont limitées à encourager l'émancipation des peuples de langue française, dont le Québec, que le président français Charles de Gaulle aurait bien aimé voir se "décoloniser » du Canada. Sa célèbre déclaration à l'hôtel de ville de Montréal le 24 juillet 1967, qui célébrait le Québec et le Canada français "libres ", a bien résonné chez Gontran Rouleau. Après le congrès de Cannes, il s'est rendu à Paris pour rencontrer l'ambassadeur du Canada, le délégué du Québec, et de Gaulle, à l'Élysée, le $1^{\mathrm{er}}$ octobre $1968^{93}$. Ce dernier s'est d'ailleurs réjoui de l'ambition du Richelieu de s'installer en France et l'a incité à élargir son territoire à tous les peuples de langue française. La Francophonie émergente avait l'avantage de rallier des nationalistes de plusieurs tendances, ce qui a permis à Rouleau, un fédéraliste, d'établir un bon rapport avec Jean-Marc Léger, un indépendantiste qui venait d'être chargé de la mise sur pied du Secrétariat permanent de l'Agence de coopération

91 Procès-verbal de la neuvième réunion du Conseil d'administration », 22-23 janvier 1971, p. 5, CRCCF, Fonds RI C76/2/7.

92 Trang Phan et Michel Guillou, Francophonie et mondialisation : histoire et institutions des origines à nos jours, avec le concours de Corine Djistera et al., et la collaboration de Sarah Boukri et al., Paris, Éditions Belin, 2011.

93 Gontran Rouleau, «Accueil chaleureux à l'Élysée », La Revue Richelieu, mai-juin 1969 , p. 43. 
culturelle et technique (ACCT) à Paris ${ }^{94}$. Léger a applaudi cette "modernisation" du Richelieu et lui a promis une subvention annuelle à hauteur de 50000 \$, même si, faute de moyens, elle se limiterait à $5000 \$ 95$.

Ce nouvel intérêt du Richelieu pour la France et la Francophonie a aussi captivé nombre de dignitaires canadiens, dont l'ambassadeur du Canada en France, Jules Léger, et le ministre de l'Éducation du Québec, Jean-Guy Cardinal ${ }^{96}$. Le ministère des Affaires intergouvernementales du Québec a offert 10000 \$ pour l'expansion du mouvement en France en 1969, la Délégation lui a fourni des contacts et le Consulat du Canada à Marseille a rangé différents objets en réserve pour les futurs cercles là-bas. Pierre Trudeau voyait aussi en l'expansion du Richelieu " une œuvre éminemment louable [...] favorisant de sorte la bonne entente et la coopération internationales ${ }^{97}$ ", mais le ministère des Affaires extérieures ainsi que l'Agence canadienne de développement international (ACDI), fondée en 1970, se sont abstenus de soutenir les efforts du Richelieu pendant ces années ${ }^{98}$. En outre, cet intérêt pour la Francophonie mondiale a emballé les membres de toute allégeance politique venant du Québec et des milieux minoritaires. Ces derniers ont d'ailleurs représenté la moitié des 900 délégués nord-américains au congrès de Cannes et effectué

94 "Procès-verbal de la sixième réunion du Conseil d'administration ", 7 novembre 1969, p. 4, CRCCF, Fonds CRO, C117-1/2/7.

95 " Procès-verbal de la troisième réunion ", 26-27 mars 1971, p. 6 ; « $1^{\text {er }}-23$ mars ", [mars 1972], p. 16; "Procès-verbal d'une réunion du comité d'expansion ", 4 janvier 1974, p. 75, CRCCF, Fonds RI, C76/3/1 et C76/3/3.

96 "Éclatant succès du congrès Richelieu ", 10 octobre 1968, p. 2, APRI, dossier "Communiqués de presse 1968-1976 "; "Réunion du comité de régie ", 18 décembre 1969 , p. 4 ; "Procès-verbal de la sixième réunion du Conseil d'administration ", 6-7 octobre 1972, p. 2, CRCCF, Fonds RI, C76/2/5.

97 Pierre Elliott Trudeau, "Message du premier ministre aux Richelieu », La Revue Richelieu, vol. 20, n 7 (décembre 1970), p. 3, CRCCF, Fonds CRO, C1172/10/19.

98 «État des revenus et dépenses», 31 décembre 1972, CRCCF, Fonds RI, C76/3/3. 
cinq des plus importantes contributions financières à l'expansion du Richelieu à l'étranger pendant la période ${ }^{99}$.

Pourtant, cet enthousiasme d'apparence quasi consensuelle pour l'élargissement du Richelieu à la Francophonie entière n'a pas favorisé l'émergence, telle qu'imaginée par Gontran Rouleau, d'une hiérarchie d'appartenances équilibrée entre les communautés locales, régionales, nationales et mondiale. Cet effort d'agrandir « la famille » canadiennefrançaise, avouait-il, constituait avant tout une « adaptation [nécessaire] au monde moderne ${ }^{100}$ " pour parvenir à mieux résister à l'hégémonie culturelle anglo-américaine. Son successeur à la présidence, le Franco-Ontarien Gaston Beaulieu, renchérirait même que " chacun se sent[ait] obligé de se couvrir d'une déclaration à caractère universel ${ }^{101}$ ». Quelques cercles se sont opposés à la mondialisation du Richelieu, certains membres y voyant une " trahison » de "l'esprit " canadien-français, celui qui avait animé ses fondateurs, entreprise seulement pour gagner des pâturages plus verdoyants. Pourtant, il apparaissait trop tard pour les dirigeants de faire marche arrière, la mondialisation étant " un mouvement irréversible " qui engageait l'organisme à " maintenir le rythme de [se]s travaux sinon les accentuer $^{102}$ ". L'idéologie mondialisante devenait une norme incontestable à un point tel que, déjà en 1971-1972, certains membres osaient même qualifier le « souci de la sauvegarde du caractère ethnique des Canadiens français ${ }^{103}$ " de rétrograde et peu adapté aux aspirations des jeunes, " ouverts ${ }^{104}$ ", pour qui la langue se réduisait à un « moyen

99 «Rapport des sommes versées au fonds d'expansion », 20 octobre 1972, CRCCF, Fonds RI, C76/3/3.

${ }^{100}$ Gontran Rouleau, dans " 1968 sera l'année internationale ", Le Richelieu, vol. 18, $\mathrm{n}^{\circ} 2$ (mars 1968), p. 2, CRCCF, Fonds CRO, C117-2/10/18.

101 Gaston Beaulieu, «Pérégrinations d'un président », Le Richelieu, mai-juin 1969, p. 16-17.

102 "Procès-verbal de la sixième réunion du Conseil d'administration », 6-7 octobre 1972, p. 3, CRCCF, Fonds RI, C76/2/5.

103 Paul Dumont, dans "Vie et survie du Richelieu International : rapport de l'atelier ", [octobre 1970], p. 2, CRCCF, Fonds RI, C76/2/7.

104 "Congrès Richelieu aux Maritimes ", La Revue Richelieu, vol. 20, n 4 (juilletaoût 1970), p. 8, CRCCF, Fonds CRO, C117-2/10/19; "Procès-verbal de la 
de communication international » qui pouvait faire " rayonner ${ }^{105}$ " l'individu à l'étranger, selon le directeur général G.-Mathias Pagé. La volonté de rupture se trouvait aussi chez l'administrateur Louis-J. Roy, qui voulait substituer au courant " nationaliste, conservateur, [et] sur la défensive " d'antan, une ouverture sur le monde qui permettrait au Richelieu d'accepter « les gens de toutes les religions, donc de toutes les idéologies ${ }^{106}$ ». Ainsi, les membres pourraient devenir de véritables « citoyens du monde ». Si certains évoquaient " la fierté nationale d'exporter une formule, un produit canadienfrançais " à l'étranger, les dirigeants faisaient des pieds et des mains pour empêcher ses membres de "se satisfaire du repli sur soi ${ }^{107}$ ".

Potentiellement exagérée, cette autocritique voulait aussi circonscrire les critiques de l'expansion sinueuse et parfois décevante dans la Francophonie. Depuis quelques années, le Richelieu avait envoyé des délégués pour sillonner l'Europe francophone et imaginait un archipel de cercles en Europe francophone, de Brest à Bruxelles et de Perpignan à Aoste, mais n'avait pas les moyens de ses ambitions. Par ailleurs, les service clubs Lions et Rotary avaient déjà recruté plusieurs membres potentiels depuis leur installation en France vingt ans plus tôt. Le Richelieu s'est plutôt tourné vers les anciens membres de la Table Ronde, une fraternité d'origine médiévale qui excluait ses membres à leur $40^{\mathrm{e}}$ anniversaire ${ }^{108}$. La stratégie s'est avérée payante dans plusieurs localités, dont à Lille (France), où 15 des 25 membres fondateurs du cercle Richelieu étaient des anciens de la Table Ronde ${ }^{109}$.

réunion du Conseil d'administration ", 13 février 1970, p. 2, CRCCF, Fonds RI, C76/2/7.

105 G.-Mathias Pagé, "Expansion et Jeunesse : vie et survie du Richelieu ", La Revue Richelieu, vol. 21, nº 1 (janvier-février 1971), p. 2, CRCCF, Fonds CRO C117-2/10/19.

106 Louis-J. Roy, «Éditorial », La Revue Richelieu, mars-avril 1971, APRI, vol «Vie Richelieu 1946-1980».

107 "Comité des œuvres ", 24 novembre [1971], p. 2, CRCCF, Fonds RI, C76/3/1. 108 "Rapport de l'agent de liaison », 15 juin 1970, p. 5, CRCCF, Fonds RI, C76/2/7.

109 "Rapport de l'agent de liaison », 27 avril 19[71], p. 2, CRCCF, Fonds RI, $\mathrm{C} 76 / 3 / 1$. 
À quelques occasions, certains membres du Rotary ont quitté leur club pour se rapprocher de leurs " cousins [canadiens-français] ${ }^{110}$ " au Richelieu, mais les gains ont été modestes. Entre 1968 et 1972, seuls neuf clubs ont été fondés en France. Vu le rythme plus élevé des affiliations de clubs en milieu minoritaire nord-américain, leur poids au Richelieu est passé de 32 à $34 \%$, la proportion des cercles québécois a diminué de 65 à $61 \%$ et les clubs outre-mer ne représentaient que $5 \%$ de l'ensemble ${ }^{111}$. Malgré l'ambition des promoteurs de la mondialisation, le coût élevé des missions à l'étranger (178 000 \$ en quatre ans, dont $58 \%$ provenaient des agences publiques $\left.{ }^{112}\right)$ et leurs faibles retombées à court terme $(44000 \$$ en nouvelles cotisations en deux ans) ont suffi pour qu'en 1973 une assemblée réachemine la moitié des sommes affectées à l'expansion aux " cadres traditionnels ", une curieuse expression pour désigner, sans le dire, le Canada français ${ }^{113}$.

Ironiquement, c'est à ce moment que le mouvement commençait à se doter d'une notoriété à l'étranger. On avait évoqué la possibilité d'affilier des groupes d'hommes noirs francisés « dans les principales capitales ${ }^{114}$ " de l'Afrique francophone, sans toutefois mettre en ouvre un plan pour veiller à sa réalisation. À l'automne 1969, le Richelieu a été stupéfait d'apprendre qu'un groupe à Kinshasa, en République démocratique du Congo, souhaitait former un cercle Richelieu. Le siège social a donc demandé à ce que le promoteur, Pierre-Honoré Masunga, un directeur d'école, confirme ses bonnes intentions puis

110 Marcel Koessler, « Procès-verbal de la quatrième réunion du Conseil d'administration ", 14 février 1969, CRCCF, Fonds RI, C76/2/6.

111 "Liste des clubs Richelieu ", mai 2011, p. 6-8, APRI, vol. «Base de données électroniques".

112 "Analyse de la rentabilité du programme d'expansion », [1974], Archives privées du Club Richelieu Edmunston, dossier " Réseau ".

113 "Vingt-troisième [...] assemblée générale », 16 février 1973, p. 6, CRCCF, Fonds RI, C76/3/1.

${ }^{114}$ Lettre de Gontran Rouleau à G.-Mathias Pagé, 8 juillet 1969, p. 5, CRCCF, Fonds RI, C76/2/6. 
l'a nommé agent de liaison adjoint pour l'Afrique ${ }^{115}$. Sur le continent, les service clubs avaient traditionnellement rassemblé des expatriés européens et une poignée symbolique d'Africains. Les idéaux d'ouverture et d'antiracisme semblant faire leur chemin au Canada français, le conseil d'administration a préconisé la fondation de « clubs en Afrique avec des Africains ${ }^{116}$ ». Il s'agirait de cercles intellectuels et caritatifs fondés par des Africains auxquels pourraient ensuite se joindre des expatriés belges, canadiens ou français. Devant dignitaires et ambassadeurs, une première remise de charte à Kinshasa le 3 juillet 1970 a voulu confirmer la " place pour tous les éléments francophones de l'univers ${ }^{117}$ " dans ce mouvement renouvelé. Les fondateurs du club ont cependant précisé que leur soutien à l'" enfance malheureuse " serait réalisé " sans " le secours d' " autrui ${ }^{118}$ ". Après ces premières fréquentations toutefois, les écarts de richesse entre l'Afrique et l'Occident poseraient des défis presque insurmontables au développement de rapports équitables et à l'expansion du Richelieu sur le continent.

C'est ainsi que, pour reprendre l'expression de Joseph Yvon Thériault, le projet du Canada français " a eu la vie dure " pendant ces années. La Révolution tranquille au Québec, l'autonomisation des minorités de langue française au niveau provincial ainsi que l'émergence d'une conscience par rapport aux espaces francophones de la planète ont laissé fort peu de place à toute réflexion axée sur les réalités communes de ceux qu'on appelait jusqu’à tout récemment les Canadiens français. Leurs particularismes régionaux et leurs

115 Lettre de G.-Mathias Pagé à l'Ambassade du Congo, 4 novembre 1969; Lettre de Jean-Baptiste Mbila à G.-Mathias Pagé, 19 novembre 1969; " Liste des membres », 5 mai 1970, APRI, vol. "Clubs », dossier « 07164 Mobutu ".

116 «Atelier des mandataires : district n ${ }^{\text {os }}$ 9, 10 et $11 », 13$ février 1970, p. 2, CRCCF, Fonds RI, C76/3/1.

117 Robert Fournier, dans "Richelieu Mobutu de Kinshasa ", La Revue Richelieu, vol. 20, n 5 (septembre-octobre 1970), p. 7, CRCCF, Fonds CRO, C1172/10/19.

118 «Au Club R/ Mobutu », 3 juillet 1970, p. 1-2, 4, APRI, vol. «Clubs », dossier «07164 Mobutu». 
divergences respectives ont occupé presque la totalité de l'espace qui restait pour dialoguer sur le potentiel d'un avenir collectif. Par ailleurs, entre une scission nationale et une forme de mondialisation, le fédéralisme renouvelé a proposé, grâce aux subventions du Secrétariat d'État, l'adhésion des individus de langue française à un Canada uni d'un océan à l'autre, avec peu d'intérêt pour le recul de la culture et de la langue dans certains milieux, ainsi que les aspirations particulières du peuple francophone enraciné au Québec et dans sa périphérie. Du côté des subventions de l'Agence de coopération culturelle et technique et des ambitions internationales des peuples de langue française, elles ont permis d'accentuer les (parfois rares) traits communs que partageaient ces derniers. Certains membres québécois se sont probablement mis à penser qu'ils avaient plus en commun avec les Français qu'avec les Acadiens, par exemple. Par conséquent, l'espoir de Gontran Rouleau de voir émerger une complémentarité entre les projets locaux, nationaux et mondiaux ne se réaliserait pas. En éclipsant les « raisons communes » du Canada français, on créait un vide qui serait comblé par les manifestations régionales et les projets francophones plus axés sur la diversité et les déplacements que sur l'autodétermination et l'enracinement.

\section{L'effritement du projet canadien-français (1973-1982)}

L'effritement du projet canadien-français était donc bel et bien enclenché. Les allusions à la solidarité canadienne-française ou aux réalités partagées se sont faites rares entre 1973 et 1982, même au Richelieu qui demeurait, en réalité, un organisme essentiellement canadien-français. La progression du terme "francophone " a été fulgurante au point de détrôner celui de " canadien-français » comme vocable prédominant, du moins à l'ouest du Québec ${ }^{119}$. En octobre 1976, Jean-Jacques Tremblay avait beau affirmer encore que le terme

119 Michel Bock, Comment un peuple oublie son nom : la crise identitaire francoontarienne et la presse française de Sudbury, 1960-1975, Sudbury, Institut francoontarien et Éditions Prise de parole, 2001. 
" canadien-français " était toujours utile pour " décrire certaines réalités qui le justifi[aient] ${ }^{120}$ ", dont la référence aux gens de souche française et aux espaces investis en Amérique du Nord, il intervenait peut-être dans un débat déjà terminé. Certains administrateurs du Richelieu continuaient de sensibiliser les membres québécois à la réalité en milieu minoritaire, mais surtout en abordant les tendances dont les Québécois devaient se méfier ${ }^{121}$. Preuve du changement de ton, si le club de Québec avait financièrement soutenu les efforts de développement institutionnel chez les minorités quinze ans plus tôt, celui-ci acceptait sans réserve le constat que le français disparaîtrait " à la prochaine génération dans l'Ouest ${ }^{122}$ ". L'appui du gouvernement du Québec au Richelieu n'a guère été substantiel pendant l'existence du Service du Canada français d'outre-frontières (19611975) ni après l'élection du Parti québécois, qui n’aurait pas tenu une promesse faite en 1976 de soutenir l'expansion du Richelieu à l'extérieur des frontières de la Belle Province ${ }^{123}$.

Du côté du Canada, le Richelieu a donc maintenu comme interlocuteurs le gouvernement fédéral et le Secrétariat d'État, qui a continué à organiser des échanges de jeunes et de tournées de conférenciers francophones dans les clubs en milieu minoritaire ${ }^{124}$. Devant cet engagement du gouvernement fédéral, qui imposait néanmoins certaines balises idéologiques aux cercles, et le désintérêt du gouvernement du Québec, le Richelieu a progressivement penché vers le fédéralisme. Le siège social a rappelé à certains cercles que le gouvernement fédéral concevait un projet national qui, malgré ses

120 Lettre de Jean-Jacques Tremblay au Droit, 21 octobre 1976, p. 2, CRCCF, Fonds JJT, P195/1/7.

121 "Richelieu! À l’aide ", Vie Richelieu, vol. 31, nº 2 (mars-avril 1981), APRI, vol. «Vie Richelieu 1981-...".

122 René Dussault, Bulletin, 3 juin 1974, p. 2, APCRQ, vol. "Bulletins ", dossier «1974».

123 « Procès-verbal de la quatrième réunion du Conseil d'administration », 28 juillet 1977, p. 64, APRI, vol. « Procès-verbaux », dossier « 1977-1978 ».

124 Ibid. 
limites profondes, comprenait les minorités de langue françaises ${ }^{125}$. D'ailleurs, le fédéralisme renouvelé amenait, indirectement, de nouveaux gains institutionnels pour l'Ontario français. Le cercle d'Ottawa continuait d'attirer l'élite professionnelle et politique francoontarienne, qui débattait vivement le partage des compétences provinciales et la reconnaissance des droits des minorités canadiennesfrançaises ${ }^{126}$. Lorsqu'une cause scolaire ou juridique survenait, on appelait les membres à « signaler leur intérêt individuel ${ }^{127}$ ", rappelant la pratique de noyautage qu'employait l'Ordre de Jacques-Cartier pour donner un élan aux revendications politiques canadiennesfrançaises ${ }^{128}$. Son penchant fédéraliste ne l'empêchait pas, toutefois, de critiquer vivement les propositions de réforme constitutionnelle qui écartaient le biculturalisme et maintiendraient le statut précaire des minorités linguistiques, et le Québec qui ne leur proposait que l'émigration ou l'acculturation ${ }^{129}$. Parmi les cercles du Sud ontarien, situés en contexte encore plus minoritaire que ceux établis dans l'Est, les préoccupations se limitaient plutôt à la réalisation de l'autonomie institutionnelle dans le secteur scolaire, qui était garante d'une certaine dualité culturelle au Canada ${ }^{130}$.

Puisqu'elle constituait déjà une entité distincte depuis trois siècles, l'Acadie se préoccupait moins de l'éloignement du Québec des minorités, d'autant plus qu'elle aussi vivait un renouveau à cette époque. Tenu à Moncton en 1973, le congrès du Richelieu a accueilli 700 délégués pour discuter du passé et de l'avenir de l'Acadie en

125 Gontran Rouleau, "Philosophie de l'expansion et le sens des différentes subventions ", Vie Richelieu, juillet-août 1974, p. 12.

126 Bulletin, 14 octobre 1971, p. 2; Bulletin, 2 décembre 1976; Bulletin, 5 mai 1977, p. 2; Bulletin, 16 décembre 1981, CRCCF, Fonds CRO, C117-1/3/8, C117-2/1/4 et C117-2/4/14.

127 Jean-Pierre Beaulne, Bulletin, 6 février 1974, p. 2, CRCCF, Fonds CRO, C117-1/2/8.

128 Bulletin, 6 février 1974, p. 2, CRCCF, Fonds CRO, C117-1/2/8.

129 Bulletin, 17 janvier 1980; Bulletin, 22 mai 1980, CRCCF, Fonds CRO, C117$1 / 3 / 5$.

130 Mémoire du Club Richelieu Toronto à la Commission sur l'unité canadienne, 29-30 novembre 1977, p. 1-4, APRI, vol. «Clubs », dossier « 07089 Toronto ». 
présence du père Clément Cormier, du député fédéral libéral Roméo LeBlanc, du consul de France et du premier ministre progressisteconservateur Richard Hatfield. Malgré le souper au homard et la prestation de Viola Légère dans La Sagouine, c'est le premier ministre Hatfield qui y a retenu l'attention quand il a reproché à la majorité anglophone sa réticence à apprendre le français ${ }^{131}$. Cependant, malgré la forte propension des cercles franco-ontariens à commenter les dossiers politiques, voire à s'y engager, le conseil d'administration a réprimandé les cercles acadiens - sous peine que leur charte soit révoquée - pour leur participation aux audiences de la Commission royale d'enquête sur l'unité canadienne (commission Pépin-Robarts) ${ }^{132}$. Cet exemple laisse croire qu'on aurait accordé des privilèges aux cercles franco-ontariens qu'on refusait aux autres.

Aux États-Unis, l'affirmation des francophones est demeurée paradoxale. Si les dernières écoles paroissiales bilingues avaient fermé leurs portes avant 1970, rendant impossible la tâche de « trouver des jeunes qui parl[ai] ent français ${ }^{133}$ " en Nouvelle-Angleterre, un engouement s'y est maintenu pour les cours de français dans les high schools publics, les célébrations de la Saint-Jean-Baptiste et les échanges avec des adolescents québécois, de même que le rêve d'une solidarité continentale ${ }^{134}$. Tenu à Hartford (Connecticut), le congrès de 1976 a rassemblé 1500 membres, dont 1200 "Canadiens francophones ${ }^{135}$ ".

131 "Procès-verbal de la sixième réunion du Conseil d'administration ", 20 septembre 1973, p. 2; Alexandre-J. Savoie, "Viens voir l'Acadie ", Vie Richelieu, vol. 23, $\mathrm{n}^{\circ} 5$ (septembre-octobre 1973), CRCCF, Fonds RI, C76/3/3 et Fonds CRO, C117-1/3/14.

132 "Procès-verbal d'une réunion », 20-21 janvier 1978, p. 23 ; « Procès-verbal d'une réunion du Conseil d'administration ", 4-5 mai 1978, p. 39, APRI, vol. "Procèsverbaux », dossier «1977-1978».

133 "Rapport d'une réunion ", 19 mai 1977, p. 18, APRI, vol. "Procès-verbaux ", dossier « 1976-1977».

134 "Président de la Commission d'échanges culturels américains et canadiensfrançais ", Vie Richelieu, vol. 25, n 1 (janvier-février 1974), p. 10, CRCCF, Fonds CRO, C117-1/3/14.

135 André Beauvais, "Un congrès exclusivement en français aux États-Unis ", Montréal-Matin, 22 octobre 1976. 
Sans doute inspiré par les progrès législatifs du français au Canada, le membre de Manchester et ancien président du mouvement et sénateur Louis-Israël Martel a déployé des efforts considérables pour amener l'administration démocrate de Jimmy Carter à reconnaître les Franco-Américains comme une " entité " et à adopter l'espagnol et le français comme langues officielles ${ }^{136}$. Toutefois, ces propositions n'ont pas connu de suite. En Floride pourtant, l'installation de commerçants et de professionnels canadiens-français a mené à l'affiliation d'un club à Hollywood en $1979^{137}$. En favorisant l'installation de compatriotes, le cercle œuvrait dans la seule région américaine où un semblant de projet canadien-français semblait toujours réussir à susciter une certaine fierté.

À l'échelle du continent, la solidarité canadienne-française suscitait encore un certain intérêt en milieu minoritaire et un peu moins au Québec. En revanche, l'« intention vitale " du Canada français, dirait Joseph Yvon Thériault, était plus souvent qu’autrement exprimée par une manifestation identitaire provinciale ou un projet fédéraliste en français ou une volonté de participer à la Francophonie mondiale. Le souvenir d'une aventure partagée, pour paraphraser encore Thériault, marquait sans conteste les gestes des administrateurs et des cercles, même s'il les rejoignait à des degrés bien variables. Avec des moyens très limités, le Richelieu a essayé de redonner souffle à des « raisons communes » en Amérique francophone et n'aurait peutêtre pas survécu s'il n'avait pas intensifié ses rapports avec les autres peuples francophones ou limité la rhétorique canadienne-française aux cercles en milieu minoritaire.

136 Bulletin, 12 mai 1978; Bulletin, 25 septembre 1979, APCRM, vol. « 19751981 »; Louis-Israël Martel, "Voyage en Louisiane », 16 décembre 1978, p. 11, APRI, vol. " Procès-verbaux », dossier «1977-1978 ".

137 Louis-Israël Martel, « Voyage Richelieu en Floride et en Louisiane », 14-28 mars 1979 ; « Rapport du directeur au Conseil d'administration », 11 avril 1980, APRI, vol. « Procès-verbaux ", dossiers « 1978-1979 » et « 1976-1983 ». 
C'est véritablement la participation à la Francophonie mondiale qui est devenue le moyen privilégié de rassembler les membres autour d'un projet commun. Situé à Ottawa, le siège social avait assez facilement accès aux bureaux des ministères fédéraux, d'où sans doute son succès à décrocher des subventions du ministère des Affaires extérieures à partir de 1973. En se dépeignant comme un interlocuteur privilégié en diplomatie francophone, le mouvement a reçu des fonds pour des projets outre-mer de l'Agence de coopération culturelle et technique à Paris et du ministère des Affaires intergouvernementales à Québec ${ }^{138}$. Malgré les engagements gouvernementaux pris à la fin des Trente Glorieuses, l'inflation et les compressions ont réduit les subventions disponibles, et donc l'ampleur des efforts à l'étranger. La somme de $154000 \$^{139}$, obtenue entre 1973 et 1981, sans compter

138 «Réunion du Comité d'expansion ", 27 août 1976, p. 65; "Comité d'expansion ", 17 février 1977, p. 16; "Rapport du Comité d'expansion », [1982], p. 8-9, APRI, vol. " Procès-verbaux ", dossiers « 1975-1976 » et "1976-1983 ".

139 "Procès-verbal de la première réunion ", 17 février 1973, p. 3; «Procès-verbal de la septième réunion ", 9 novembre 1973, p. 5-6; " Procès-verbal de la première réunion ", 3 mai 1975, p. 3, 19; « Procès-verbal. Conseil d'administration », 22 novembre 1978, p. 2, Archives privées du Club Richelieu Liège (ci-après APCRL), vol. " Procès-verbaux 1978-1979 "; "Procès-verbal de la troisième réunion du Conseil d'administration ", 12 juillet 1974, p. 14; "Programme d'expansion : état des revenus et dépenses ", 31 décembre 1973; " Procès-verbal de la neuvième réunion du Conseil d'administration », 22-23 février 1974, p. 8 ; «Rapport de la première réunion du Comité d'expansion ", 10 mai 1974, p. 11 ; "Procès-verbal de la quatrième réunion du Conseil d'administration ", 6 septembre 1974, p. 40, CRCCF, Fonds RI, C76/3/3 et C76/3/4; "Programme d'expansion : état des revenus et dépenses ", 31 décembre 1975, p. 53; "Rapport de la réunion du comité d'expansion ", 16 janvier 1976, p. 45; "Première réunion du Comité d'expansion », 5 novembre 1977, p. 2 ; G.-Mathias Pagé, « Rapport du directeur général », 3 octobre 1979, p. 70 ; « Rapport du délégué général », [janvier 1979], p. 17; «Rapport d'activités ", 13 mars-31 juillet [1981], p. 11; "Procès-verbal de la deuxième réunion du Conseil d'administration ", 4-5 décembre 1981, p. 14, 17, APRI, vol. " Procès-verbaux ", dossiers " 1975-1976 », « 1978-1979 " et «1976-1983 »; Lettre de Guy Trépanier à Marius Bujold, « Fonds 672 : Contributions au Richelieu international et au Conseil de la vie française en Amérique ", 7 janvier 1982, p. 2; Lettre de Grégoire Pagé à Marius Bujold, 19 janvier 1982, BAC, Fonds Ministère des Affaires extérieures (ci-après MAE), RG25, vol. 12572, dossier 26-6. 
le soutien de l'infrastructure diplomatique du Canada et du Québec à l'étranger, a quand même permis au Richelieu de lancer 120 projets de cercles à l'étranger ${ }^{140}$. Dès 1973 , le Richelieu était aussi l'un des deux seuls organismes, avec l'Association des universités partiellement ou entièrement de langue française, à siéger au comité consultatif de l'Agence de coopération culturelle et technique pour " assurer une coopération efficace entre l'Agence, les organisations internationales, [et] les associations internationales non gouvernementales ${ }^{141}$ ». Les rencontres tenues en Europe et en Afrique ont aussi permis à Grégoire Pagé de croiser politiciens et journalistes de plusieurs horizons, et de susciter, du coup, un intérêt pour le Richelieu dans les lieux les plus éloignés ${ }^{142}$.

Cette forme de "voisinage $[. .$.$] tant sur le plan local que sur le$ plan international ${ }^{143}$ ", a avoué le président Gérard L. Pelletier, diluait toutefois la place du national. Certes, le monde anglo-britannique avait une communauté mondiale (le Commonwealth) et la Francophonie était un " actif qui s'ajout[ait] à d'autres ${ }^{144}$ " identités régionales, mais les cercles qui refusaient d'intégrer certains aspects mondialisants à leurs traditions étaient presque ostracisés pour leur prétendu " manque d'ouverture " à l'" autre " francophone ${ }^{145}$. Ces membres récalcitrants jugeaient qu'on liquidait la conscience de la fragilité canadienne-française en Amérique en réduisant la solidarité à ce que

140 Rapport de G.-Mathias Pagé au Conseil d'administration, 4 mai 1978, p. 31; "Procès-verbal d'une réunion du Conseil d'administration ", 4-5 mai 1978, p. 39, APRI, vol. « Procès-verbaux ", dossier « 1977-1978 ".

141 "Tournée en Europe, Tunisie », 16 septembre-3 décembre 1973, p. 85, CRCCF, Fonds RI, C76/3/3.

142 "Rapport du délégué général », 6 septembre-12 décembre 1974, p. 55, CRCCF, Fonds RI, C76/3/4.

143 Gérard L. Pelletier, "Éditorial : message inaugural du nouveau président international ", Vie Richelieu, vol. 25, n 2 (mars-avril 1975), p. 3, CRCCF, Fonds CRO, C117-1/3/14.

144 "Réunion annuelle de l’assemblée générale », 28-30 mars 1974, p. 6 et 12, CRCCF, Fonds RI, C76/3/4.

145 Rapport de G.-Mathias Pagé, 3 octobre 1979, p. 69, APRI, vol. « Procès-verbaux 1978-1979». 
Grégoire Pagé appelle une simple " poignée de main entre francophones ${ }^{146}$ ". Ladministrateur Gaston Paradis résumait ainsi la difficulté de conjuguer la Francophonie et la nation canadienne-française :

S’ouvrir ainsi sur la francophonie mondiale, développer un esprit international et rechercher des moyens d'action adaptés, [nous] oblige à enrichir nos connaissances sur la francophonie, à réviser nos idées[, mais] pose évidemment aussi des problèmes nouveaux. Comment dans ces démarches rester fidèles à l'idéal des fondateurs? Comment répondre aux aspirations de la nouvelle génération au sein de laquelle nous voulons recruter? Comment sauvegarder notre identité de [C]anadiens[] français, des 7 millions que nous sommes, parmi les 200 millions de parlants français qui deviennent notre nouvelle cible ${ }^{147}$ ?

En France, la question de la Francophonie se posait dans le contexte de la construction politique et économique de l'Europe et du rétrécissement de la sphère d'influence de l'Hexagone à l'étranger ${ }^{148}$. La possibilité de redorer le blason de la France par l'entremise de la Francophonie interpellait donc les nationalistes français. La joie de redécouvrir l'Amérique française où, se surprenait-on, «il y avait encore une communauté vivante de francophones ", aurait permis à certains membres français de surmonter leur " complexe vis-à-vis des Américains ${ }^{149}$ ", selon l'un de ces membres. Si cette redécouverte les amenait à dégager des impressions superficielles de la réalité canadienne-française, la volonté des adhérents et les amitiés tissées étaient sincères ${ }^{150}$. Le mouvement a ainsi affilié des cercles dans les

146 Entrevue avec Grégoire Pagé, Ottawa (Ontario), 22 juin 2011.

147 Gaston Paradis, "Extrait du discours ", Vie Richelieu, vol. 29, n 5 (octobredécembre 1979), p. 16, CRCCF, Fonds CRO, C117-1/3/14.

148 Georges Duby (dir.), Histoire de la France des origines à nos jours, Paris, Larousse, 2006, p. 1095 et 1333.

149 Jean-Vincent de Saint-Phalle, "Propos d'un Richelieu de France », Vie Richelieu, vol. 23, n 3 (mai-juin 1973), p. 10, APRI, vol. «Vie Richelieu 1946-1980».

150 "Réunion du comité d'expansion », 25 juin 1976, p. 63, APRI, vol. "Procèsverbaux », dossier " 1975-1976 »; " Réunion annuelle de l'assemblée générale », 28-30 mars 1974, p. 12, CRCCF, Fonds RI, C76/3/4 ; "Réunion... », " Procès- 
villes portuaires de Brouage et de Saint-Malo, les lieux de naissance respectifs des explorateurs Samuel de Champlain et Jacques Cartier. Entre 1976 et 1981, certains clubs ont aussi organisé quelques échanges distincts entre jeunes cadiens, canadiens-français, français, franco-américains, martiniquais et wallons ${ }^{151}$. Le cercle de Paris était plus enclin à se voir comme un point de ralliement des francophones de la planète, vu l'adhésion de plusieurs diplomates d'Afrique et de quelques Canadiens. Il a, par exemple, pressé l'Académie française d'admettre un premier membre africain en la personne de Léopold Senghor, qui deviendrait un invité assidu du cercle après avoir été admis à l'Académie ${ }^{152}$. Cependant, il ne fallait pas creuser très loin pour éveiller des sensibilités nationales. À plusieurs reprises, les cercles français ont refusé d'intégrer les pratiques canadiennes-françaises qu'ils jugeaient vieillottes, dont la prière, le chant de ralliement ou la charité symbolique, par exemple ${ }^{153}$.

Grâce aux efforts de Belges expatriés au Québec, du Secrétariat d'État, du ministère des Affaires extérieures et de la Délégation du Québec à Bruxelles, le Richelieu s'est aussi établi en Belgique ${ }^{154}$.

verbal de la quatrième réunion du Conseil d'administration ", 28 juillet 1977, p. 62-63, APRI, vol. " Procès-verbaux ", dossier " 1977-1978 ».

151 Bulletin, 26 février 1980, APCRM, vol. " 1975-1981 »; " Procès-verbal ", 16 avril 1975; Lettre de G. Brout, 24 octobre 1977; Lettre de Léon Degeer, 5 février 1979, APCRL, vol. " Procès-verbaux ", dossiers " 1977-1978 " et " 19781980 »; «Le Richelieu à l'œuvre au Forum pour jeunes canadiens », Vie Richelieu, vol. 27, no 3 (juin-août 1977), p. 16; "Des jeunes d'ailleurs en vacance [sic] chez des Richelieu canadiens ", Vie Richelieu, vol. 30, n 4 (octobre-décembre 1980), p. 14; Rapport d'activités de Grégoire Pagé, 29 janvier-13 mars 1981, p. 12, APRI, vol. "Procès-verbaux " et "Vie Richelieu 1946-1980 ", dossier «1980-1981 »; G.-Mathias Pagé, « Projets d'accueil Richelieu », 28 mars 1973; Jean-Paul Rieux, "Carrefours-Richelieu-Jeunesse ", 17 mai 1973, CRCCF, Fonds $\mathrm{RI}, \mathrm{C} 76 / 3 / 3$; "Une expérience enrichissante : on s'en souviendra longtemps ", La Voix de l'Est, 3 avril 1973.

152 Simon-Pierre Nothomb, "Genèse du Cercle Richelieu Senghor ", courriel envoyé à l'auteur, 5 novembre 2011.

153 "Procès-verbal d'une réunion du comité de régie ", 8 juin 1973, CRCCF, Fonds RI, C76/3/3; "Tournée... », 16 septembre-3 décembre 1973, p. 82.

154 " Procès-verbal de la quatrième réunion du Conseil d'administration ", 8 juin 1973, p. 3; "Rapport de la tournée en Europe », 15-30 mai 1973, p. 30-31, 
L'organisation s'intéressait à ce royaume au moment où se produisait une crise constitutionnelle fédérale qui opposait la communauté wallonne historiquement dominante, mais en voie de désindustrialisation, et la communauté flamande, dynamique et en pleine quête d'autonomie. De fil en aiguille, la formation de partis politiques linguistiques a encouragé, dès 1970, la décentralisation progressive de l'État belge au profit de conseils régionaux et de communautés culturelles ayant chacune des compétences propres. Dans ce contexte tendu, dès sa fondation en 1974 et surtout après l'élection du Parti québécois en 1976, le cercle de Liège a accueilli plusieurs combats idéologiques entre les tenants de la souveraineté du Québec et ceux du fédéralisme canadien renouvelé. C'est ainsi que les ministres Camille Laurin, Claude Morin et René Lévesque et même la chanteuse Pauline Julien y sont passés pour faire la promotion du français comme langue officielle unique et du projet de la souverainetéassociation $^{155}$. Jean-Marc Léger leur dirait que l'autodétermination était " l'aboutissement normal ${ }^{156}$ " du destin d'un peuple. Pour sa part, le ministre des Affaires intergouvernementales du Québec, François Cloutier, aurait trouvé au "Richelieu-Liège [...] un relais utile, sinon nécessaire à la promotion de "La Belle Province" en Wallonie ${ }^{157}$ ». De son côté, l'ancien membre du Richelieu et député fédéral libéral de Cornwall (Ontario) Lucien Lamoureux a visité annuellement le cercle de Liège, à titre d'ambassadeur du Canada en Belgique à cette époque, pour rappeler non seulement l'appui du

CRCCF, Fonds RI, C76/3/3; Entrevue avec Grégoire Pagé, Ottawa (Ontario), 10 mai 2011.

155 "Un "Club Richelieu” est né à Liège », La dernière heure, 4 octobre 1974; «Visite de Pauline Julien ", 19 novembre 1974; "Visite de l'Ambassadeur du Canada ", 7 janvier 1975, p. 2; Camille Laurin, "Le docteur Camille Laurin a présenté son "livre blanc" ", Libre Belgique, [printemps 1978], APCRL, vol. " Procèsverbaux ", dossiers "1974-1976" et " 1977-1978 ".

156 Jean-Marc Léger, "Causerie prononcée à l'intention du club Richelieu, Liège », 9 mai 1979, p. 3, APCRL, vol. " Procès-verbaux ", dossier " 1978-1980 ".

157 " Assemblée générale ", 4 novembre 1976, APCRL, vol. " Procès-verbaux ", dossier «1974-1976». 
Canada à la Francophonie, mais aussi la nécessité d'inclure les minorités francophones dans toute entente constitutionnelle qui découlerait des négociations entre Ottawa et les provinces ${ }^{158}$. «Particulièrement avertis sur de tels sujets ${ }^{159}$ ", les membres du cercle de Liège n'hésitaient pas à afficher leur penchant résolument autonomiste tout en refusant de prendre position sur l'indépendance politique de leur région... ou encore celle du Québec ${ }^{160}$.

Pendant cette période, les efforts d'expansion ont aussi porté fruit dans les départements d'outre-mer (DOM) de France, où les révoltes des esclaves n'avaient débouché ni sur l'indépendance politique ni sur le renvoi des colons blancs. Malgré le niveau de développement supérieur à celui des îles voisines, l'ouverture d'une première université en 1972 et la reconnaissance officielle du créole, d'importants écarts ont perduré entre les descendants d'Afrique et ceux de la métropole ${ }^{161}$. Lors d'une mission en octobre 1974, Pagé y a trouvé des Antillais avides de tisser des liens avec le Canada français. Il espérait également que ses compatriotes fassent des DOM antillais une région touristique privilégiée ${ }^{162}$. C'est le gérant d'une succursale de la Banque royale du Canada qui a mené le processus d'affiliation visant à "mêler les races, les classes et les professions ${ }^{163}$ ", conformément au souhait du Richelieu. Entre 1976 et 1981, des

158 "L'Ambassadeur du Canada à Liège », Gazette de Liège, 17 octobre 1977, APCRL, vol. " Procès-verbaux ", dossiers « 1974-1976 " et " 1977-1978 "; " Assemblée générale ", 4 novembre 1976, p. 2, APCRL, vol. " Procès-verbaux ", dossier "1974-1976».

159 "Documentation pour discours sur cda et Francophonie : De bru à extott ", 27 janvier 1981, p. 2, BAC, Fonds MAE, RG25, vol. 12572, dossier 26-6.

160 Discours sur les relations économiques. Monsieur le Président, [printemps 1975]; "Procès-verbal : conseil d'administration ", 19 janvier 1976; "M. Outers : la Wallonie est aliénée dans un État unitaire ", Libre Belgique, 15 janvier 1976, APCRL, vol. " Procès-verbaux ", dossier « 1974-1976 ».

161 Jacques Dumont, L'amère patrie : histoire des Antilles françaises au XX' siècle, Paris, Fayard, 2010, p. 12-13, 192-199 et 234.

162 "Le Richelieu International en Haïti ", 17 mars 1973, CRCCF, Fonds RI, C76/3/3 et C76/3/4.

163 "Synthèse du compte rendu de la tournée [...] en Martinique et en Guadeloupe », 13-29 octobre 1974, p. 39 et 45, CRCCF, Fonds RI, C76/3/3 et C76/3/4. 
clubs semblables seraient fondés en Martinique, en Guadeloupe et en Guyane. On aurait bien voulu fonder des cercles avec des « jeunes dynamiques $[. .$.$] engagés dans l'avenir de leur pays { }^{164}$ » dans les principales villes d'Haïti, mais la résistance d'un groupe montréalais, qui y voyait " un appui direct à la dictature ${ }^{165}$ " de Jean-Claude Duvalier si le mouvement devait s'y établir, et surtout l'instabilité du pays ont conduit les missions de 1973, 1977 et 1980 à des échecs ${ }^{166}$.

On avait aussi tâté le terrain en Afrique après la fondation du cercle de Kinshasa, mais il est demeuré le seul jusqu'à la formation, à Dakar en 1973, d'un cercle composé de gens d'affaires locaux et de diplomates expatriés. Grégoire Pagé s'est rendu à Abidjan, à Beyrouth, au Caire, à Niamey et à Tunis, par exemple, pour explorer les possibilités d'affiliation ${ }^{167}$, mais sans résultat satisfaisant pendant la décennie vu les limites à la liberté d'association, au droit de parole et à la distribution de la richesse. On aurait plus de succès pendant la décennie 1980. Les possessions françaises dans l'océan Pacifique se sont aussi retrouvées dans la mire du Richelieu et du ministère des Affaires extérieures pendant un moment en 1981, mais ces efforts, comme tant d'autres, n'ont pas connu de suite ${ }^{168}$.

Entre 1973 et 1982, on a fondé une dizaine de clubs par année, dont la moitié au Québec, signe de l'intérêt pour la nouvelle mission

164 "Mission des Antilles », avril 1977, p. 26, APRI, vol. « Procès-verbaux », dossier «1976-1977».

165 "Le Richelieu International en Haïti », 17 mars 1973; "Synthèse du compte rendu de la tournée [...] en Martinique et en Guadeloupe ", 13-29 octobre 1974, p. 39 et 45, CRCCF, Fonds RI, C76/3/3 et C76/3/4.

166 Lettre de Grégoire Pagé à Denis Potvin, 28 janvier 1981, BAC, Fonds MAE, RG25, vol. 12572, dossier 26-6.

167 "Procès-verbal d'une réunion du comité d'expansion ", 28 avril 1973, p. 2; "Rapport de la mission », 3-30 juin 1974, p. 29; Bulletin, 22 août 1979, CRCCF, Fonds RI, C76/3/3 et Fonds CRO, C117-1/3/4.

168 Denis Turcotte, «La Francophonie océanienne : situation actuelle et évolution future ", [1981], p. 7; Document sans titre, [1981]; Lettre d'Armand Moudave de Mézières à Mathias Pagé, $1^{\text {er }}$ octobre 1981, BAC, Fonds MAE, RG25, vol. 12572, dossier 26-6; Lettre de Grégoire Pagé à Marius Bujold, 19 janvier 1982, BAC, Fonds MAE, RG25, vol. 12572, dossier 26-6. 
du Richelieu. Ensuite, douze cercles en France, neuf en Ontario, sept en Acadie, six en Martinique et en Guadeloupe, cinq en Belgique, quatre en Nouvelle-Angleterre, trois dans l'Ouest canadien, un en Floride et un au Sénégal se sont joints au mouvement ${ }^{169}$. C'est ainsi que le Richelieu avait, en 1982, mis le pied sur trois continents.

\section{$\operatorname{sen} 20$}

Entre 1944 et 1982, les manœuvres ont été nombreuses, surtout pendant la décennie 1960, pour maintenir un certain caractère canadien-français, ainsi que le Richelieu, à flot. En trois temps, nous avons vu comment l'organisme a établi un réseau reliant les îlots de l'Amérique française, au moment où cette solidarité par-delà les frontières était indispensable au nationalisme canadien-français. Ces efforts ont toutefois été confrontés à la volonté moderniste de la Révolution tranquille, qui a laissé peu de place à la nation organique (sans frontières définies) d'autrefois. Le Richelieu a donc redoublé d'efforts pour adopter des réformes administratives, pour écarter certains cercles qui voulaient québéciser le mouvement et pour s'ouvrir au monde francophone, contribuant ainsi à solidifier un nouvel archipel, celui-ci de la Francophonie.

Assez curieusement, le Richelieu a continué de nourrir un certain esprit canadien-français après 1967, partiellement par l'entremise de ses efforts pour développer une Francophonie mondiale. L'organisme a associé son esprit fondateur à une tradition administrative, celle des "cadres traditionnels ", un héritage envers lequel plusieurs membres de longue date se sentaient toujours redevables. Toutefois, le mouvement semblait largement impuissant à contrer les tendances lourdes de l'époque, soit la marginalisation de l'intérêt des Québécois pour les minorités canadiennes-françaises et acadienne et la progression constante des projets canadien et francophones, tous subventionnés par les gouvernements du Canada et du Québec et par l'Agence de coopération culturelle et technique. Le développement de rapports

169 "Liste des clubs Richelieu ", 4 février 2013, p. 8-12, APRI, vol. " Base de données électroniques ». 
sociaux, parfois superficiels, entre peuples francophones a suscité un engouement sincère chez les nouveaux et les anciens membres du Richelieu. Franco-Ontariens, Québécois, Sénégalais et Wallons confondus ont pris part à l'édification de ce projet, même si leur expérience locale colorait plus souvent qu'autrement leurs regards et leurs motivations. La promotion de la langue et des cultures françaises dans le monde a insufflé un dynamisme nouveau au Richelieu, comparable en intensité, mais plus ambigu sur le plan des objectifs concrets, au nationalisme canadien-français qui avait animé ses fondateurs.

Le Canada français s'est-il perdu entre la provincialisation des discours nationalistes et l'émergence d'un discours francophone mondial? Il a été coincé, avons-nous dit, bien involontairement entre la fragmentation des identités et la mondialisation des cultures, deux tendances fortes des mutations nationales des décennies 1960 et 1970. Pourtant, le pari qu'a fait le mouvement Richelieu, en contribuant à ériger un nouvel échelon de solidarité francophone, a aussi contribué à affaiblir le nationalisme canadien-français dans un des rares lieux où les Québécois et les minorités francophones participaient d'un même mouvement. En somme, la mondialisation des référents identitaires a eu un rôle à jouer, parallèlement aux autres tensions qui touchaient les débats constitutionnels et les rapports OttawaQuébec et minorités-majorité, dans l'énigmatique démantèlement du Canada français. 


\section{Annexe}

Effectifs et poids des cercles du Richelieu par région ${ }^{170}$

\begin{tabular}{|l|c|c|c|c|c|c|}
\hline AnNÉE & QuÉBEC & $\begin{array}{c}\text { AMÉrique } \\
\text { FRANÇAISE }\end{array}$ & ANTILLES & EuROPE & AfriQuE & $\begin{array}{c}\text { Total des } \\
\text { MEMBrES }\end{array}$ \\
\hline 1949 & $69 \%$ & $31 \%$ & & & & 1000 \\
\hline 1954 & $76 \%$ & $24 \%$ & & & & 3400 \\
\hline 1960 & $76 \%$ & $24 \%$ & & & & 6000 \\
\hline 1969 & $65 \%$ & $35 \%$ & & & & 6500 \\
\hline 1973 & $61 \%$ & $34 \%$ & & $5,0 \%$ & $0,5 \%$ & 6400 \\
\hline 1978 & $62 \%$ & $32 \%$ & $0,5 \%$ & $5,0 \%$ & $0,5 \%$ & \\
\hline 1982 & $55 \%$ & $33 \%$ & $1,7 \%$ & $9,0 \%$ & $1,5 \%$ & 6500 \\
\hline 1986 & $52 \%$ & $33 \%$ & $1,5 \%$ & $13 \%$ & $1,2 \%$ & 7100 \\
\hline 1990 & $51 \%$ & $32 \%$ & $1,6 \%$ & $15 \%$ & $1,0 \%$ & 7600 \\
\hline 1994 & $49 \%$ & $33 \%$ & $1,7 \%$ & $16 \%$ & $1,0 \%$ & 6900 \\
\hline 2013 & $45 \%$ & $32 \%$ & $1,7 \%$ & $20 \%$ & $2,1 \%$ & 4300 \\
\hline
\end{tabular}

170 Ibid., p. 1-12. 